\title{
Effects of multi-domain interventions in (pre)frail elderly on frailty, functional, and cognitive status: a systematic review
}

This article was published in the following Dove Press journal:

Clinical Interventions in Aging

24 May 2017

Number of times this article has been viewed

\author{
Lenore Dedeyne' \\ Mieke Deschodt ${ }^{2-4}$ \\ Sabine Verschueren ${ }^{5}$ \\ Jos Tournoy ${ }^{1,3}$ \\ Evelien Gielen ${ }^{1,3}$ \\ 'Department of Clinical and \\ Experimental Medicine, ${ }^{2}$ Department \\ of Public Health and Primary Care, \\ KU Leuven - University of Leuven, \\ Leuven, Belgium; ${ }^{3}$ Department of \\ Geriatric Medicine, University \\ Hospitals Leuven, Leuven, Belgium; \\ ${ }^{4}$ Department of Public Health, \\ Institute of Nursing Science, \\ University of Basel, Basel, Switzerland; \\ ${ }^{5}$ Department of Rehabilitation \\ Sciences, KU Leuven - University \\ of Leuven, Heverlee, Belgium
}

Background: Frailty is an aging syndrome caused by exceeding a threshold of decline across multiple organ systems leading to a decreased resistance to stressors. Treatment for frailty focuses on multi-domain interventions to target multiple affected functions in order to decrease the adverse outcomes of frailty. No systematic reviews on the effectiveness of multi-domain interventions exist in a well-defined frail population.

Objectives: This systematic review aimed to determine the effect of multi-domain compared to mono-domain interventions on frailty status and score, cognition, muscle mass, strength and power, functional and social outcomes in (pre)frail elderly ( $\geq 65$ years). It included interventions targeting two or more domains (physical exercise, nutritional, pharmacological, psychological, or social interventions) in participants defined as (pre)frail by an operationalized frailty definition.

Methods: The databases PubMed, EMBASE, CINAHL, PEDro, CENTRAL, and the Cochrane Central register of Controlled Trials were searched from inception until September 14, 2016. Additional articles were searched by citation search, author search, and reference lists of relevant articles. The protocol for this review was registered on PROSPERO (CRD42016032905).

Results: Twelve studies were included, reporting a large diversity of interventions in terms of content, duration, and follow-up period. Overall, multi-domain interventions tended to be more effective than mono-domain interventions on frailty status or score, muscle mass and strength, and physical functioning. Results were inconclusive for cognitive, functional, and social outcomes. Physical exercise seems to play an essential role in the multi-domain intervention, whereby additional interventions can lead to further improvement (eg, nutritional intervention).

Conclusion: Evidence of beneficial effects of multi-domain compared to mono-domain interventions is limited but increasing. Additional studies are needed, focusing on a well-defined frail population and with specific attention to the design and the individual contribution of mono-domain interventions. This will contribute to the development of more effective interventions for frail elderly.

Keywords: nutrition, supplement, exercise, cognition, hormone, social, vulnerable, older adults

\section{Introduction}

Frailty is a late-life syndrome that results from reaching a threshold of decline across multiple organ systems. ${ }^{1}$ Because frailty leads to excess vulnerability and reduced ability to maintain homeostasis, frail elderly are predisposed to functional deficits, comorbidity, and mortality. ${ }^{1,2}$

Despite a lack of international consensus on the definition of frailty, two conceptual definitions are commonly used. ${ }^{3,4}$ First, Fried et al introduced the frailty phenotype and described frailty based on the presence of five physical characteristics: unintentional
Correspondence: Evelien Gielen Department of Geriatric Medicine, University Hospitals Leuven, Herestraat 49/7003 35, Leuven, Belgium

Tel +32 I6 340931

Email evelien.gielen@uzleuven.be 
weight loss, weakness, exhaustion, slow gait, and low physical activity. ${ }^{5,6}$ Subjects are considered robust (no criteria present), prefrail ( 1 or 2 criteria present), or frail (3-5 criteria present). ${ }^{5}$ Second, the frailty index of Rockwood and Mitnitski defines frailty as an accumulation of heterogeneous deficits identified by a comprehensive geriatric assessment. ${ }^{7}$ The index reflects the proportion of present deficits to the total number of potential deficits, to determine whether a patient is robust ( $\leq 20 \%)$, prefrail $(20 \%-35 \%)$, or frail $(\geq 35 \%))^{7,8}$ The frailty index represents a broader scope of frailty, including cognitive, social, and psychological components, next to the physical characteristics. Although the frailty syndrome includes multiple domains, physical frailty (and more specifically musculoskeletal frailty) is seen as the main component of frailty. ${ }^{2,9}$

Depending on the frailty definition and evaluation tool, frailty prevalence ranges between $4.0 \%$ and $59.1 \%$ in community-dwelling people aged $>65$ years. ${ }^{10}$ As the population ages, frailty represents increasingly important public health concerns and has an incremental effect on health expenditures (additional $\pm € 1500$ /frail person/year). ${ }^{11,12}$ Because of the major clinical and economic burden, it is critical to find efficient, feasible, and cost-effective interventions to prevent or slow down frailty in order to avoid or diminish the adverse outcomes and maintain or improve quality of life. ${ }^{1,9}$

Frailty is possibly reversible or modifiable by interventions. ${ }^{13-16}$ Previous research on nonpharmacological interventions such as physical exercise and nutritional interventions showed promising effects on frailty status, functional outcomes, and cognitive outcomes. ${ }^{17-19}$ These interventions can be combined with each other or with other (eg, pharmacological, hormonal, or cognitive) therapies to prevent or treat frailty. ${ }^{20}$ As frailty results from reaching a threshold of decline in different physiological systems, the approach to address frailty should act on multiple domains. ${ }^{13,21}$

Previous overviews of multi-domain interventions only examined combinations of exercise and nutritional interventions. ${ }^{22}$ Also, studies combining more than two domains were not in their scope of interest. ${ }^{23}$ Other reviews focused on other populations (eg, sarcopenia or obesity) $)^{22,24-26}$ or included studies that used no diagnostic tool to determine the frail population ${ }^{27,28}$ or used poor search criteria for (pre) frail participants (eg, only one keyword in database search). ${ }^{29}$ Because of the limitations of existing reviews as well as to include information from recent studies, a systematic review was conducted, aiming to provide an overview of the effects of controlled multi-domain interventions in (pre)frail people aged $\geq 65$ years on frailty status and score, cognition, muscle mass strength and power, and functional and social outcomes.

\section{Methodology}

The review protocol was registered in the PROSPERO database (CRD42016032905) and was reported in accordance with the PRISMA guidelines. ${ }^{30}$

\section{Search methods}

First, a systematic literature search was conducted in PubMed, EMBASE, CINAHL, PEDro, CENTRAL, and the Cochrane Central register of Controlled Trials from inception of the database until September 14, 2016, to ensure comprehensive article retrieval. The search strategy was developed for PubMed (Figure S1) and then translated for use in other databases (available upon request). The literature search was limited to articles published in English, Dutch, French, or German and excluded case reports, letters, and editorials. Second, additional studies were searched by handsearching reference lists, citations, and other publications from first and last authors from relevant papers retrieved in the first search.

\section{Inclusion and exclusion criteria}

The inclusion criteria are as follows: 1) randomized controlled trials, quasi-experimental studies, or prospective or retrospective cohort studies with control groups; 2) testing of a multi-domain intervention to prevent or treat frailty in people aged $\geq 65$ years; 3 ) classification in terms of (pre) frailty status according to an operationalized definition; and 4) primary outcomes including one or more of the following: frailty status or score, muscle mass, strength or power, physical functioning, and cognitive or social outcomes.

A multi-domain intervention was defined as an intervention that intervenes in at least two different domains, including exercise therapy (Ex), nutritional intervention (supplementation of proteins [NuP], supplementation of vitamins and minerals [NuVM], milk fat globule membrane $[\mathrm{NuMF}]$, or nutritional advice [NuAd]), hormone (Hor), cognitive (Cog) or psychosocial (PS) interventions. Studies that did not compare groups in view of the delivered multidomain intervention were excluded.

\section{Study selection}

Identification of potentially relevant papers based on title and abstract was conducted by one reviewer (LD). Thereafter, the full texts of all potentially relevant abstracts were examined 
for eligibility. In case of inconclusiveness, a second reviewer (JT) was consulted to discuss eligibility. In case of disagreement, consensus was sought between the reviewers or involvement of a third reviewer (EG) was asked.

\section{Critical appraisal}

Risk of bias in the individual studies was assessed by the methodological index for nonrandomized studies (MINORS). The following 12 MINORS criteria were evaluated by two independent researchers (LD and MD): clearly stated aim, inclusion of consecutive patients, prospective collection of data, endpoints appropriate to the aim of the study, unbiased assessment of the study endpoint, follow-up period appropriate to the aim of the study, loss to follow-up $<5 \%$, prospective calculation of the study size, adequate control group, contemporary groups, baseline equivalence of groups, and adequate statistical analyses. ${ }^{31}$ Each criterion was scored 0 (not reported), 1 (reported but inadequate), or 2 points (reported and adequate), resulting in a total quality score ranging from 0 (low quality) to 24 (high quality).

\section{Data extraction and synthesis}

The following data were extracted from the included studies: study characteristics (aim, country, design, and setting), participant characteristics (age and gender), frailty diagnostic tool, characteristics of multi-domain interventions (duration, content, frequency, intensity, follow-up moments, and compliance), characteristics of intervention and control groups (number of participants and loss to follow-up), frailty status or score, cognition, muscle mass, strength or power, functional and social outcomes, and quality-of-life measurements. Data on effect measures (mean \pm standard deviation or median [10th-90th percentile]) of the included studies were extracted up to 1 year after the intervention. Data were extracted by one researcher (LD) and checked by a second reviewer (EG or JT). Disagreements were resolved by discussion between the two reviewers.

The primary outcomes were frailty status, cognition, muscle mass, muscle strength, and power and functional outcomes. Secondary outcomes are quality of life, social involvement, psychosocial well-being, and depression and subjective health. The effects between intervention groups were reported, as this study focuses on the effect of multi-domain interventions compared to mono-domain interventions. No effects over time within individual groups were reported. No meta-analysis was performed due to high heterogeneity of the study interventions and outcomes.

\section{Results Study selection}

Figure 1 visualizes the study selection process based on the PRISMA flowchart. ${ }^{30}$ The literature search yielded 5,500 publications. To assess the eligibility of the articles, full texts were read, and 200 articles were excluded with reasons (Table S1). Overall, 24 articles reporting on twelve individual studies met the study eligibility criteria and were included in this systematic review. ${ }^{32-55}$

\section{Study characteristics}

Table 1 summarizes the study characteristics. Five studies were conducted in Europe, ${ }^{33,46,50,53,54}$ two in the USA, ${ }^{41,42}$ and five in Asia. ${ }^{32,43-45,55}$ Eleven studies ${ }^{32,33,41-46,50,53,54}$ had a randomized controlled design and one study ${ }^{55}$ had a randomized crossover design. The studies ranged in sample size from 31 to $246 .^{41,45}$ The duration of the intervention varied between 12 weeks ${ }^{32,43,44,53,55}$ and 9 months. ${ }^{46}$ Five studies included a follow-up moment at 3-9 months after the intervention. ${ }^{32,43-46}$ Nine of the twelve studies were dated from 2010 onwards. . $^{32,42-45,50,53-55}$

One study did not report participant setting, ${ }^{55}$ whereas all others recruited participants living in the community. Three studies included only women. ${ }^{42-44}$ To select (pre)frail participants, the Fried frailty phenotype criteria were most frequently used $(n=8)$, often modified or in combination with additional criteria. ${ }^{32,42-45,50,54,55}$ Two studies used the frailty definition of Chin A Paw et al, ${ }^{33,46}$ one study ${ }^{41}$ used the physical performance test score of Reuben and Siu, ${ }^{90}$ and one study examined (pre)frailty by the Survey of Health, Ageing and Retirement in Europe Frailty Instrument for Primary Care (SHARE-FI). ${ }^{53}$ Studies included only frail, ${ }^{33,42,43,46,54}$ moderate frail, ${ }^{41}$ only prefrail, ${ }^{44}$ or both prefrail and frail phenotypes. ${ }^{32,45,50,53,55}$

\section{Quality of the study}

The total methodological quality scores of the included studies ranged from 16 "moderate" 33,41 to 23 "excellent" 43 (Table 2). Only six studies prospectively calculated the sample size. ${ }^{43,45,50,53-55}$ Baseline differences between intervention and control groups were observed in four studies ${ }^{32,46,53,54}$ and were not reported in one study. ${ }^{41}$

\section{Characteristics of the multi-domain intervention programs}

Table 3 summarizes the interventions of the twelve included studies. Nine studies combined two domains in their 


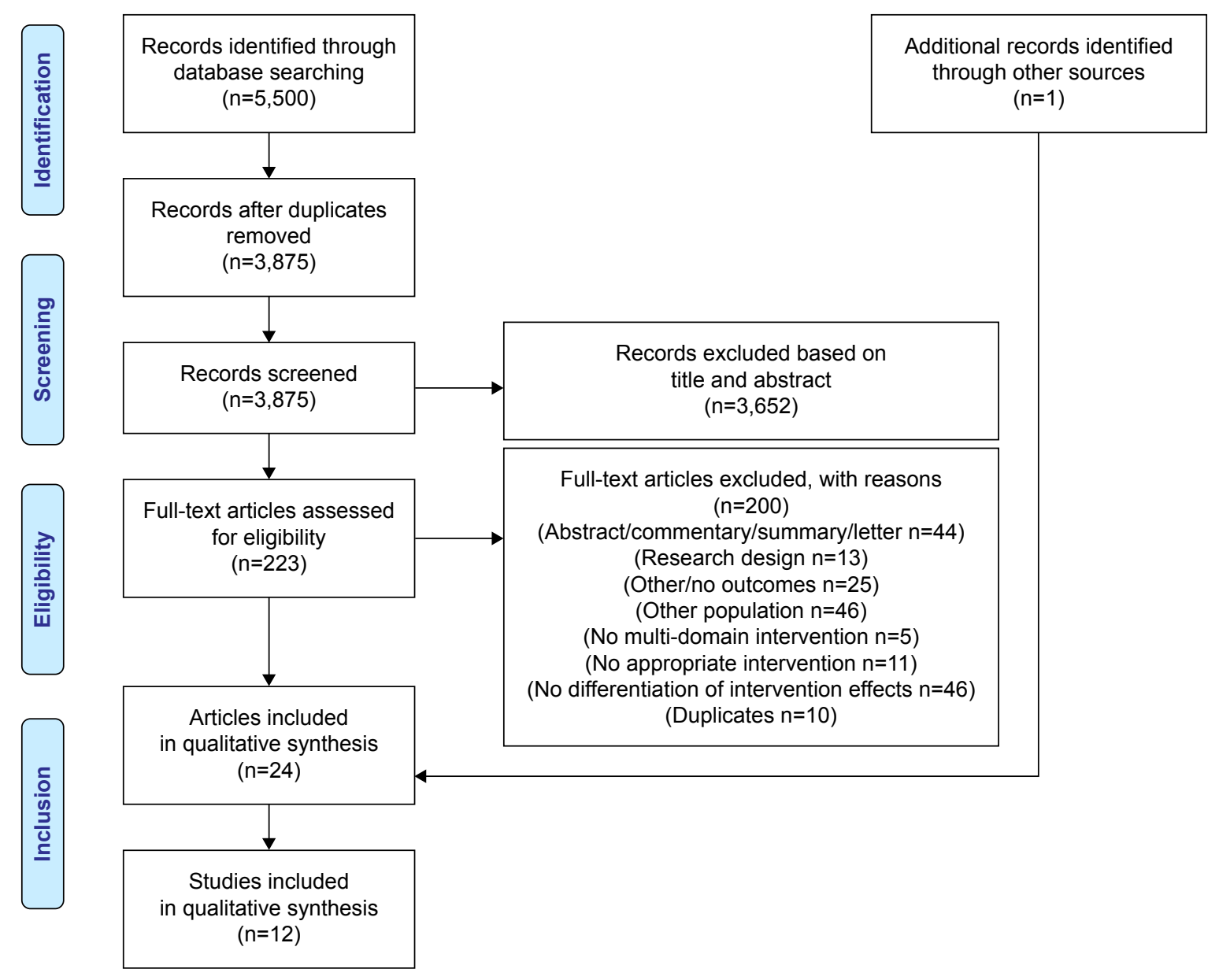

Figure I Flow chart of study selection process.

interventions. Thereof, eight combined a nutritional and physical activity intervention, ${ }^{33,43,44,46,50,53-55}$ and one combined hormone therapy with physical activity intervention. ${ }^{41}$ The remaining three studies combined three interventions, of which one study combined exercise, nutritional, and hormone intervention, ${ }^{42}$ one study combined exercise and nutritional intervention with psychotherapy, ${ }^{32}$ and one study combined exercise, nutritional, and cognitive interventions. ${ }^{45}$ All the studies $(\mathrm{n}=12)$ included an exercise intervention, and all but one study ${ }^{42}$ included a strength training component. Three of the twelve studies included an exercise intervention with only a strength training component, ${ }^{41,50,53}$ whereas the other nine studies included a multicomponent exercise intervention (at least two of following components: endurance and/or strength and/or balance and/or flexibility). All but one study ${ }^{41}$ included a nutritional intervention: four studies provided nutritional advice, ${ }^{32,44,46,53}$ seven studies provided nutritional supplementation, ${ }^{33,42,43,45,50,54,55}$ and one study provided both nutritional advice and supplementation. ${ }^{54}$ Compliance was reported in nine studies: ${ }^{32,33,42,45,46,50,53-55}$ compliance to the exercise intervention ranged from $47.3 \%{ }^{54}$ to $\geq 95 \%,{ }^{55}$ and compliance to the nutritional intervention ranged from $73 \%{ }^{46}$ to $100 \% .^{55}$

\section{Impact of the intervention strategies on frailty Change in frailty status and frailty score}

Five studies assessed the impact of a multi-domain intervention on frailty status (frail, prefrail, or robust) and/or frailty score (0-5 points) (Table 4). . $^{32,43,45,53,54}$ Postintervention, four studies found a significantly improved frailty status or score in the multi-domain intervention groups $\left(\mathrm{Ex}+\mathrm{NuMF}^{43} ; \mathrm{Ex}+\right.$ $\mathrm{NuP}+\mathrm{NuVM}+\mathrm{Cog}^{45} ; \mathrm{Ex}+\mathrm{NuAd}+\mathrm{PS}$ and $\mathrm{Ex}+\mathrm{NuAd}^{32} ;$ $\mathrm{Ex}+\mathrm{NuAd}+\mathrm{NuVM}^{54}$ ) compared to mono-domain intervention groups or control group. ${ }^{32,43,45,54}$ One study found no significant difference on SHARE-FI score between an Ex + NuAd and a social support intervention. ${ }^{53}$ At 4 months follow-up, in one study, larger significant improvements were maintained in groups with an exercise intervention irrespective of their additional nutritional intervention $(\mathrm{Ex}+\mathrm{NuMF}$ 


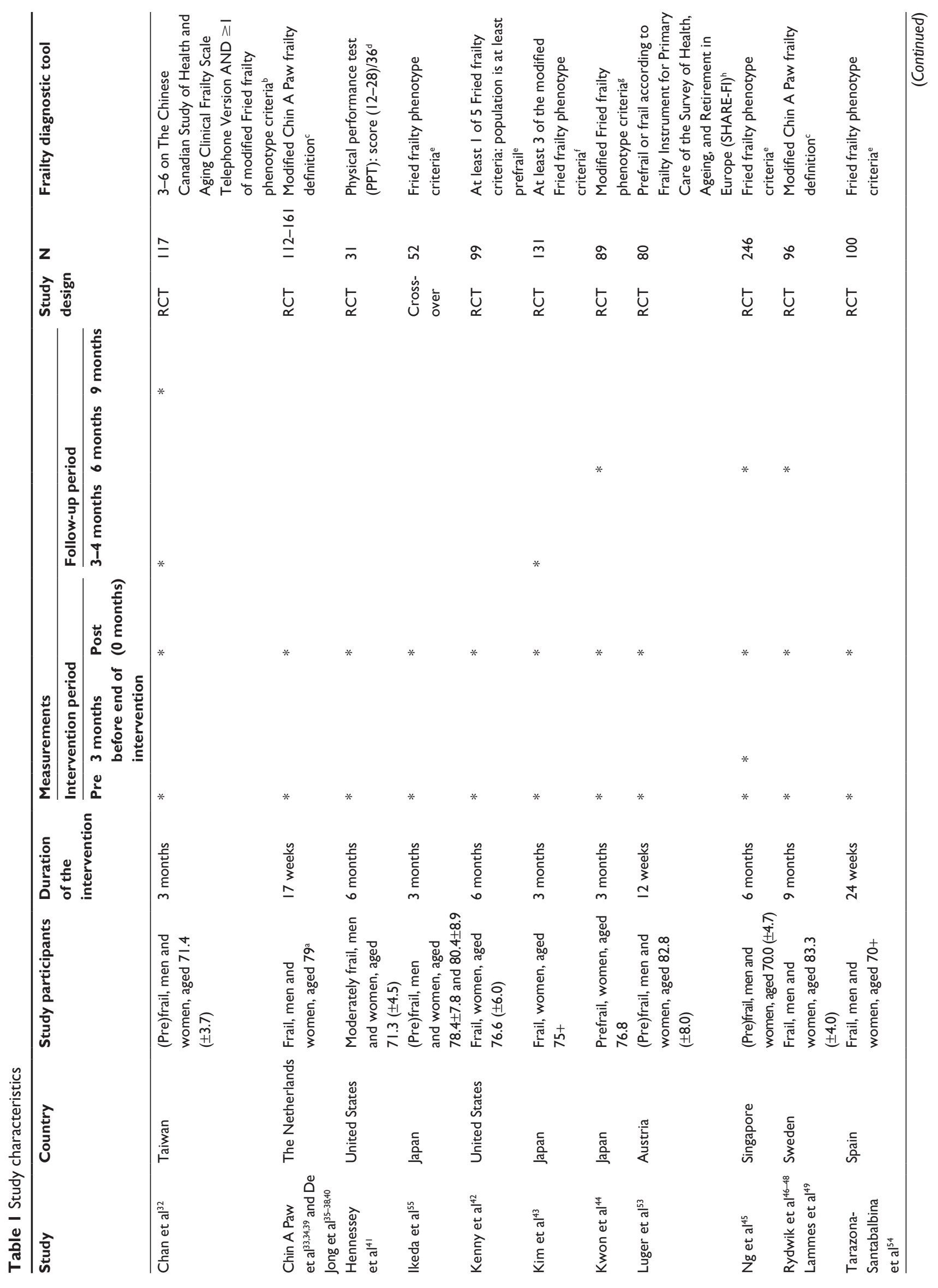




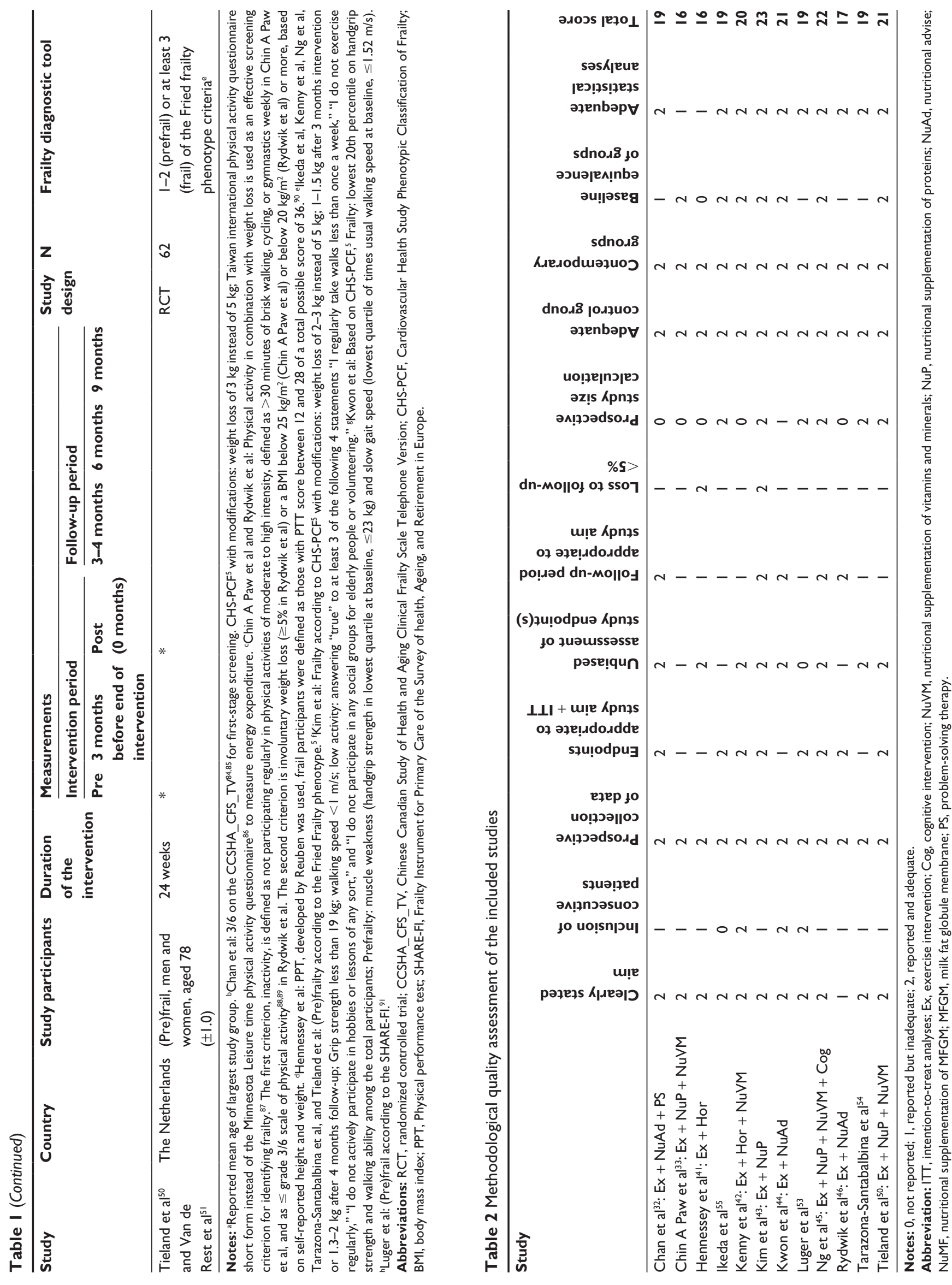


and $\mathrm{Ex}+\mathrm{NuP}$ intervention groups compared to $\mathrm{NuP}$ for frailty status, and compared to NuP and NuMF for frailty score). ${ }^{43}$ In another study, participants of the $\mathrm{Ex}+\mathrm{NuAd}( \pm \mathrm{PS})$ intervention did not maintain its significant larger improvement of frailty status at 3 months follow-up compared to control or a PS interventions. ${ }^{32}$ At 6 months follow-up, the multi-domain $\mathrm{Ex}+\mathrm{NuP}+\mathrm{NuVM}+\mathrm{Cog}$ intervention of $\mathrm{Ng}$ et al showed a significantly improved frailty status and score (higher frailty reduction odds ratio compared to control group) compared to the mono-domain interventions. ${ }^{45}$ Overall, multi-domain interventions showed significantly larger improved frailty status and score in four studies compared to mono-domain or control interventions. ${ }^{32,43,45,54}$

\section{Impact of the intervention strategies on the elements of the frailty phenotype}

Fried et $\mathrm{al}^{5}$ described frailty in the Cardiovascular Health Study (CHS). More specifically, the Phenotypic Classification of Frailty (CHS-PCF) ${ }^{5}$ includes five components: unintentional weight loss, weakness, exhaustion, slow gait, and low physical activity. ${ }^{5,6}$ In the following section, effects of multi-domain interventions in frail elderly on these components are described.

\section{Change in muscle mass (unintentional weight loss)}

Seven studies examined muscle mass after a multi-domain intervention (Table 5). First, Tieland et al found that adding a protein and mineral supplementation to an exercise intervention significantly improved appendicular and total muscle mass post-intervention. ${ }^{50}$ Chin A Paw et al found a significantly improved muscle mass by an exercise intervention combined with a protein, vitamin, and mineral supplementation intervention compared to protein, vitamin, and mineral supplementation. ${ }^{36}$ Kenny et al found that adding a hormonal dehydroepiandrosterone intervention to an exercise and vitamin and mineral supplementation increased total (but not appendicular) lean mass post-intervention. ${ }^{42}$ The other four studies found no significant differences between multidomain and mono-domain interventions. No significant effect was found of adding a milk fat globule membrane (MFGM) or protein supplementation to an exercise intervention. ${ }^{43}$ Psychosocial intervention (problem-solving therapy) with or without Ex + NuAd intervention compared to no psychosocial intervention or an $\mathrm{Ex}+\mathrm{NuAd}$ intervention with or without a PS intervention compared to no Ex + NuAd intervention had also no significant effect on muscle mass. ${ }^{32}$ Also, the combination of Ex $+\mathrm{NuAd}$ did not alter muscle mass significantly compared to Ex or NuAd alone or control group. ${ }^{46}$
Tarazona-Santabalbina et al found that adding exercise to nutritional advice and vitamin and mineral supplementation intervention did not significantly improve muscle mass. ${ }^{54}$

\section{Change in muscle strength (weakness)}

Muscle strength was examined in nine studies, combining Ex + $\mathrm{NuP},{ }^{43,55} \mathrm{Ex}+\mathrm{NuMF},{ }^{43} \mathrm{Ex}+\mathrm{NuP}+\mathrm{NuVM},{ }^{33,50} \mathrm{Ex}+\mathrm{NuAd},{ }^{44,46}$ $\mathrm{Ex}+\mathrm{NuP}+\mathrm{NuVM}+\mathrm{Cog},{ }^{45} \mathrm{Ex}+\mathrm{NuVM}+\mathrm{Hor},{ }^{42} \mathrm{Ex}+\mathrm{Hor}^{41}$ (Table 6). One study found that adding protein supplementation to a strength and balance exercise intervention significantly improved leg press strength and knee extension strength but not for hip abduction strength and rowing. ${ }^{55}$ Two studies examined the effect of 3 months Ex + NuAd. ${ }^{44,46}$ Rydwik et al found in both Ex $+\mathrm{NuAd}$ and Ex groups a significantly improved lower (compared to NuAd) and upper (elbow) (compared to control group) muscle strength post-intervention, but not in shoulder muscle strength. ${ }^{46}$ Kwon et al found no significant differences between Ex $+\mathrm{NuAd}$ and other intervention groups post-intervention. ${ }^{44}$ At 6 months follow-up, they found significantly declined muscle strength in the Ex $+\mathrm{NuAd}$ group compared to the control group. ${ }^{44}$ The interventions $\mathrm{Ex}+\mathrm{NuP}$ $+\mathrm{NuVM}+\mathrm{Cog}$, Ex alone, and Cog alone showed significantly improved lower muscle strength both post-intervention and at 6 months follow-up compared to the control group. ${ }^{45}$ Finally, Kenny et al found that adding a hormone intervention to an exercise and vitamin and mineral supplementation significantly improved lower but not upper muscle strength. ${ }^{42}$ Some studies found no significant differences between multi-domain interventions and mono-domain interventions: there was no significant effect in adding an MFGM (NuMF) ${ }^{43}$ or protein supplementation (with vitamin/mineral supplementation) ${ }^{50}$ or hormone intervention ${ }^{41}$ to an exercise intervention. Chin A Paw et al found no significantly improved muscle strength by an exercise intervention combined with a protein, vitamin, and mineral supplementation intervention compared to protein, vitamin, and mineral supplementation or by combined exercise and nutritional intervention compared to single exercise or no intervention. ${ }^{33}$

\section{Exhaustion}

The exhaustion component of the phenotypical frailty definition cannot be covered in the "Results" section as none of the included articles described the effect of multi-domain interventions on exhaustion.

\section{Gait speed (slow gait)}

Seven studies measured gait speed (Table 7A). Overall, two studies found more improved gait speed in multi-domain 
Table 3 Intervention characteristics

\begin{tabular}{|c|c|c|c|c|}
\hline \multirow[t]{2}{*}{ Study } & \multicolumn{2}{|l|}{ Exercise intervention } & \multicolumn{2}{|l|}{ Nutritional intervention } \\
\hline & Content & Frequency & Content & Frequency \\
\hline Chan et $\mathrm{al}^{32}$ & $\begin{array}{l}\text { Warm up (brisk walk, stretching major } \\
\text { joints and muscles), resistance training, } \\
\text { postural control activities, and balance } \\
\text { training, cool down (relaxation) } \\
\text { Resistance weights: rubber band and } \\
\text { water (0.6-I L); intensity not reported } \\
=\text { Ex }\end{array}$ & $\begin{array}{l}3 \times / \text { week } \\
60 \mathrm{~min}\end{array}$ & $\begin{array}{l}\text { Nutritional consultation: } \\
\text { possibility to ask dietary } \\
\text { questions, assess dietary } \\
\text { compliance } \\
=\mathrm{NuAd}\end{array}$ & $\begin{array}{l}\text { During exercise } \\
\text { session } \\
(3 \times / \text { week })\end{array}$ \\
\hline $\begin{array}{l}\text { Chin A Paw et al } \\
\text { and de Jong } \\
\text { et } \mathrm{al}^{33-40}\end{array}$ & $\begin{array}{l}\text { Warm-up (walking and exercise- } \\
\text { to-music routines), skills training } \\
\text { (strength training with } 450 \mathrm{~g} \text { wrist } \\
\text { and ankle weights, speed, flexibility, } \\
\text { coordination, endurance) to perform } \\
\text { and sustain motor actions (reaching, } \\
\text { throwing, catching, kicking, etc), cool } \\
\text { down (stretching and relaxation) } \\
\text { Gradually increased intensity: train } \\
\text { at intensity between } 6 \text { and } 8 \text { on a } \\
\text { I0-point perceived exertion scale } \\
=\text { Ex }\end{array}$ & $\begin{array}{l}2 \times / \text { week } \\
45 \mathrm{~min}\end{array}$ & $\begin{array}{l}\text { Supplementation: fruit and dairy } \\
\text { products enriched with vitamins } \\
\text { and minerals at } 25 \%-100 \% \text { of } \\
\text { recommended dietary allowance } \\
=\mathrm{NuP}+\mathrm{NuVM}\end{array}$ & $\begin{array}{l}\text { I fruit and I dairy } \\
\text { product/day }\end{array}$ \\
\hline Hennessey et $\mathrm{al}^{41}$ & $\begin{array}{l}\text { Warm-up, low impact graded } \\
\text { resistance training, cool down. Knee } \\
\text { extension and ankle dorsiflexion: } \\
\text { WI-5: } 20 \%-60 \% \text { I RM; W6-9: } \\
80 \%-90 \% \text { I RM; W I0-17 \& WI8-25: } \\
60 \%-95 \% \text { retested I RM } \\
\text { Plantar flexion: weights 5\%-10\% of } \\
\text { body weight or quadriceps strength } \\
\text { and progress } 2 \%-5 \% \text { every } 2 \text { weeks } \\
=\text { Ex }\end{array}$ & $\begin{array}{l}3 \times / \text { week } \\
60 \mathrm{~min}\end{array}$ & & \\
\hline Ikeda et $\mathrm{al}^{55}$ & $\begin{array}{l}\text { Muscle strength exercise (intensity: } \\
30 \% \text { of MVC; } 3 \text { sets of } 20 \text { repetitions), } \\
\text { aerobic exercise (intensity I } 2 \text { on BRP), } \\
\text { balance exercise, cool down } \\
=\text { Ex }\end{array}$ & $2 \times /$ week & $\begin{array}{l}6 \mathrm{~g} \text { Branched-chain amino } \\
\text { acid (BCAA) supplementation } \\
(\text { I,560 mg BCAA; I,440 mg } \\
\text { essential AA) } \\
=\text { NuP }\end{array}$ & $\begin{array}{l}2 \times / \text { week within } \\
10 \text { min before } \\
\text { exercise }\end{array}$ \\
\hline Kenny et $\mathrm{al}^{42}$ & $\begin{array}{l}\text { Yoga (lvengar): breathing exercises, } \\
\text { postures focusing on balance and } \\
\text { stretching, relaxation; progressive } \\
\text { difficulty OR } \\
\text { Chair aerobics: commercially available } \\
\text { tapes of moderate aerobic effort, } \\
\text { increasing difficulty from week 4-6 } \\
\text { onwards } \\
\text { Intensity not reported } \\
=\text { Ex }\end{array}$ & $\begin{array}{l}2 \times / \text { week } \\
90 \min \end{array}$ & $\begin{array}{l}\text { All participants: } 630 \mathrm{mg} \text { calcium } \\
\text { and } 400 \mathrm{IU} \text { cholecalciferol } \\
=\mathrm{NuVM}\end{array}$ & $\mathrm{I} \times /$ day \\
\hline Kim et $\mathrm{a}^{43}$ & $\begin{array}{l}\text { Warm-up, progressive strengthening } \\
\text { exercises (with Thera bands, } \\
\text { increasing repetitions), balance and } \\
\text { gait training, cool down } \\
\text { Moderate intensity: } 12-14 \text { on BRP } \\
=\text { Ex }\end{array}$ & $\begin{array}{l}2 \times / \text { week } \\
60 \mathrm{~min}\end{array}$ & $\begin{array}{l}6 \text { pills with each } 167 \mathrm{mg} \\
\text { MFGM ( } 2 \text { I \% protein; } 44 \% \text { fat; } \\
26.5 \% \text { carbohydrate, } 33.3 \% \\
\text { phospholipids) } \\
=\text { NuMF }\end{array}$ & $\mathrm{I} \times /$ day morning \\
\hline
\end{tabular}




\begin{tabular}{ll}
\hline Other intervention & \\
\hline Content & Frequency \\
\hline - Problem-solving therapy: & $2 \times /$ month \\
solve problems contributing & problem-solving \\
to mood-related conditions, & therapy \\
increase self-efficacy & \\
- All participants: educational & \\
booklet on frailty, healthy & \\
diets, exercise protocols, & \\
and self-coping strategies & \\
$=$ PS &
\end{tabular}

\section{Control intervention}

Content

Non-Ex and NuAd and non-

PS: how much they read the

booklet and how well they

complied with suggested

diet and exercise protocols

described in educational

booklets

Exercise: social program

(lectures, social activities,

and crafts)

Home visit for supply of fresh

food products

Nutritional: same foods as

intervention group but not

enriched (= NuP)

I $\times / 2$ weeks
90 min
$I \times / 2$ weeks
I fruit and I dairy
product/day

Frequency

Ix/month

$\times /$ day

Hormone: placebo injections

$0.0025-0.0037 \mathrm{mg} / \mathrm{kg}$

$=$ Hor
IX/day

\section{Compliance}

Ex + Nu: $18 / 55$

participants attended at least $50 \%$ of the

36 intervention sessions

PST: 17/57 completed the 6 courses

Ex: attendance: $90 \%$

(range 47\%-100\%)

$\mathrm{Nu}$ : high compliance:

percentage of participants

with at least one

deficiency decreased from

$61 \%$ to $15 \%$

Control: attendance: $80 \%$

(range 50\%-100\%)

Not reported
Nutritional: maltodextrin

(MD)

Hormone: placebo

IX/day

$2 \times /$ week within $10 \mathrm{~min}$ before exercise

Ex: compliance rate $\geq 95 \%$ Nu: compliance rate $100 \%$

Ex: $73.1 \% \pm 24.2 \%$

adherence

DHEA/placebo:

$88.9 \% \pm 22.4 \%$ adherence
Nutritional: placebo with similar shape, taste and texture but whole milk powder instead of MFGM (26.3\% protein;

$25.2 \%$ fat; $39.5 \%$ carbohydrate, $0.286 \%$ phospholipids)

$=\mathrm{NuP}$ 
Table 3 (Continued)

\begin{tabular}{|c|c|c|c|c|}
\hline \multirow[t]{2}{*}{ Study } & \multicolumn{2}{|l|}{ Exercise intervention } & \multicolumn{2}{|l|}{ Nutritional intervention } \\
\hline & Content & Frequency & Content & Frequency \\
\hline Kwon et $\mathrm{al}^{44}$ & $\begin{array}{l}\text { Warm up, stretching exercises, } \\
\text { exercises aiming to increase muscle } \\
\text { strength and balance capability, } \\
\text { cool down } \\
\text { Strength training: one set of } \\
5 \text { repetitions progressing to I set } \\
\text { of } 10 \text { repetitions } \\
=\text { Ex }\end{array}$ & $\begin{array}{l}1 \times / \text { week } \\
60 \mathrm{~min}\end{array}$ & $\begin{array}{l}\text { - Nutritional education: lecture } \\
\text { - Cooking classes: using food } \\
\text { ingredient rich in protein and } \\
\text { vitamin D } \\
=\text { NuAd }\end{array}$ & I $\times /$ week \\
\hline Luger et $\mathrm{al}^{53}$ & $\begin{array}{l}\text { Warm-up and strength training } \\
(6 \text { exercises, } 2 \text { sets of } 15 \text { repetitions } \\
\text { until muscular exhaustion) + physical } \\
\text { education } \\
=\text { Ex }\end{array}$ & $\begin{array}{l}2(-3) \times / \text { week } \\
30 \mathrm{~min}\end{array}$ & $\begin{array}{l}\text { Dietary discussions: how to } \\
\text { enrich food with protein, } \\
\text { recipes, healthy for life plate } \\
=\text { NuAd }\end{array}$ & $2 \times /$ week \\
\hline $\mathrm{Ng}$ et $\mathrm{al}^{45}$ & $\begin{array}{l}\text { Strength and balance training. } \\
\text { Resistance training integrated with } \\
\text { functional tasks } \\
\text { Gradually increasing intensity: } \\
\text { I set of } 8-\text { I } 5 \text { RM or } 60 \%-80 \% \text { of } \\
\text { I0 RM, starting with }<50 \% \text { I RM. } \\
\text { Balance training involving functional } \\
\text { strength, sensory input, and added } \\
\text { attentional demand } \\
=\text { Ex }\end{array}$ & $\begin{array}{l}2 \times / \text { week } \\
90 \mathrm{~min}\end{array}$ & $\begin{array}{l}\text { Supplementation: } \\
\text { - Fortisip Multi Fibre } \\
\quad \text { (I2 g protein) } \\
\text { - Iron } \\
\text { - Folate } \\
\text { - Vit B6, Vit BI2 } \\
\text { - Calcium and Vit D } \\
=\text { NuP + NuVM }\end{array}$ & $\mathrm{I} \times /$ day \\
\hline $\begin{array}{l}\text { Rydwik et } \mathrm{al}^{46-48} \\
\text { and } \\
\text { Lammes et } \mathrm{al}^{49}\end{array}$ & $\begin{array}{l}\text { Warm-up (aerobic training), } \\
\text { progressive muscle strength training } \\
\text { (WI-2: 60\% I RM; at W3: } 80 \% \text { I RM; } \\
\text { I RM measurement was repeated } \\
\text { at W6 and WI0), Qigong balance } \\
\text { training, cool down } \\
\text { Weights: } 10 \%-20 \% \text { of body weight } \\
=\text { Ex }\end{array}$ & $\begin{array}{l}2 \times / \text { week } \\
60 \mathrm{~min}\end{array}$ & $\begin{array}{l}\text { - Individual dietary counseling } \\
\text { based on baseline food record } \\
\text { data } \\
\text { - Group session covering topics } \\
\text { as nutritional needs for the } \\
\text { elderly with serving of well- } \\
\text { balanced snack } \\
=\text { NuAd }\end{array}$ & $\begin{array}{l}1 \times \text { in total } \\
60 \text { min } \\
5 \text { sessions }\end{array}$ \\
\hline $\begin{array}{l}\text { Tarazona- } \\
\text { Santabalbina et } \text { al }^{54}\end{array}$ & $\begin{array}{l}\text { Proprioception and balance exercises, } \\
\text { aerobic training (initially } 40 \% \text { of } \\
\text { maximum heart rate to } 65 \% \text { ), strength } \\
\text { (initially } 25 \% \text { of } \\
\text { I RM to } 75 \% \text { ), stretching } \\
=\text { Ex }\end{array}$ & $\begin{array}{l}5 \times / \text { week } \\
65-70 \mathrm{~min}\end{array}$ & $\begin{array}{l}\text { Nutritional information of } \\
\text { the optimal energy intake, } \\
\text { the requirement to ensure } \\
\text { a minimal protein intake of } \\
0.8 \mathrm{~g} / \mathrm{kg} \text { body weight. Calcidol } \\
\text { levels }<30 \mathrm{ng} / \mathrm{mL} \text { : calcidol } \\
\text { loading dose. } \\
\text { Supplementation of I,200 mg } \\
\text { calcium and } 800 \mathrm{IU} \text { calciferol } \\
=\text { NuAd + NuVM }\end{array}$ & IX/day \\
\hline $\begin{array}{l}\text { Tieland et } \mathrm{al}^{50} \\
\text { and }\end{array}$ & $\begin{array}{l}\text { Warm up, resistance exercises } \\
\text { at increasing intensity }(50 \%\end{array}$ & $2 \times /$ week & $\begin{array}{l}\text { Supplementation: } 250 \mathrm{~mL} \\
\text { protein supplemented beverage }\end{array}$ & $\begin{array}{l}2 \times / \text { day: after } \\
\text { breakfast and }\end{array}$ \\
\hline Van de Rest et $\mathrm{al}^{51}$ & $\begin{array}{l}\text { [10-15 repetitions] - to } 75 \% \\
\text { [8-10 repetitions] of I RM). } \\
\text { I RM measurement was repeated } \\
\text { after } 4,8,12,16 \text {, and } 20 \text { weeks } \\
\text { of training } \\
=\text { Ex }\end{array}$ & & $\begin{array}{l}\text { with I } 5 \text { g protein, } 7.1 \text { g lactose, } \\
0.5 \mathrm{~g} \text { fat, } 0.4 \text { g calcium } \\
=\mathrm{NuP}+\mathrm{NuVM}\end{array}$ & after lunch \\
\hline
\end{tabular}

Abbreviations: RM, repetition maximum; Vit, vitamin; rhGH, recombinant human growth hormone; sc, subcutaneous; MFGM, milk fat globule membrane; HRT, hormone replacement therapy; CE, conjugated estrogens; MPA, medroxyprogesterone acetate; DHEA, dehydroepiandrosterone; min, minutes; Hor, hormonal intervention; Ex, exercise intervention; Cog, cognitive intervention; NuVM, nutritional supplementation of vitamins and minerals; NuP, nutritional supplementation of proteins; NuAd, nutritional advise; NuMF, nutritional supplementation of MFGM; PS, problem-solving therapy; BRP, Borg Rate of Perceived Exertion scale; MVC, maximal voluntary contraction; $\mathrm{Cl}$, confidence interval; $\mathrm{W}$, week; $\mathrm{AA}$, amino acid. 


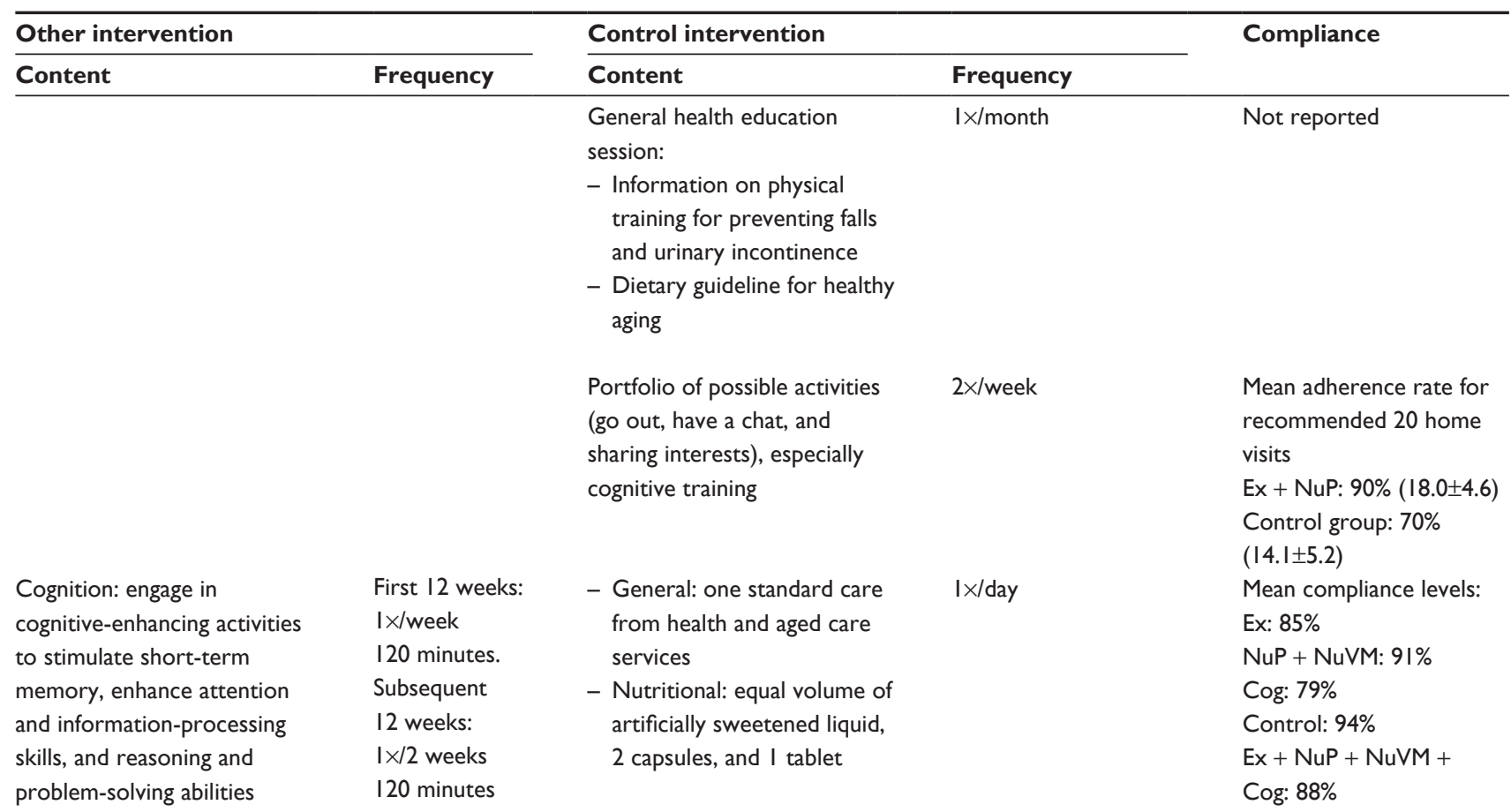

General advice regarding physical activity and nutrition for the elderly

Nutritional: identical

IX/day

$38.7 \%-55.7 \%)$
Nutritional: placebo drink

without protein, $7.1 \mathrm{~g}$ lactose,

$0.4 \mathrm{~g}$ calcium
Ex: $47.3 \%(95 \% \mathrm{Cl}$
Ex: mean compliance rate was $65 \%(4 \%-100 \%)$

$\mathrm{Nu}$ : mean compliance rate was $73 \%(20 \%-100 \%)$

\author{
Mean adherence rate for \\ visits \\ $\pm 4.6)$ \\ (I4.I \pm 5.2$)$ \\ Ex: $85 \%$ \\ Cog: $79 \%$ \\ M
}

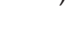




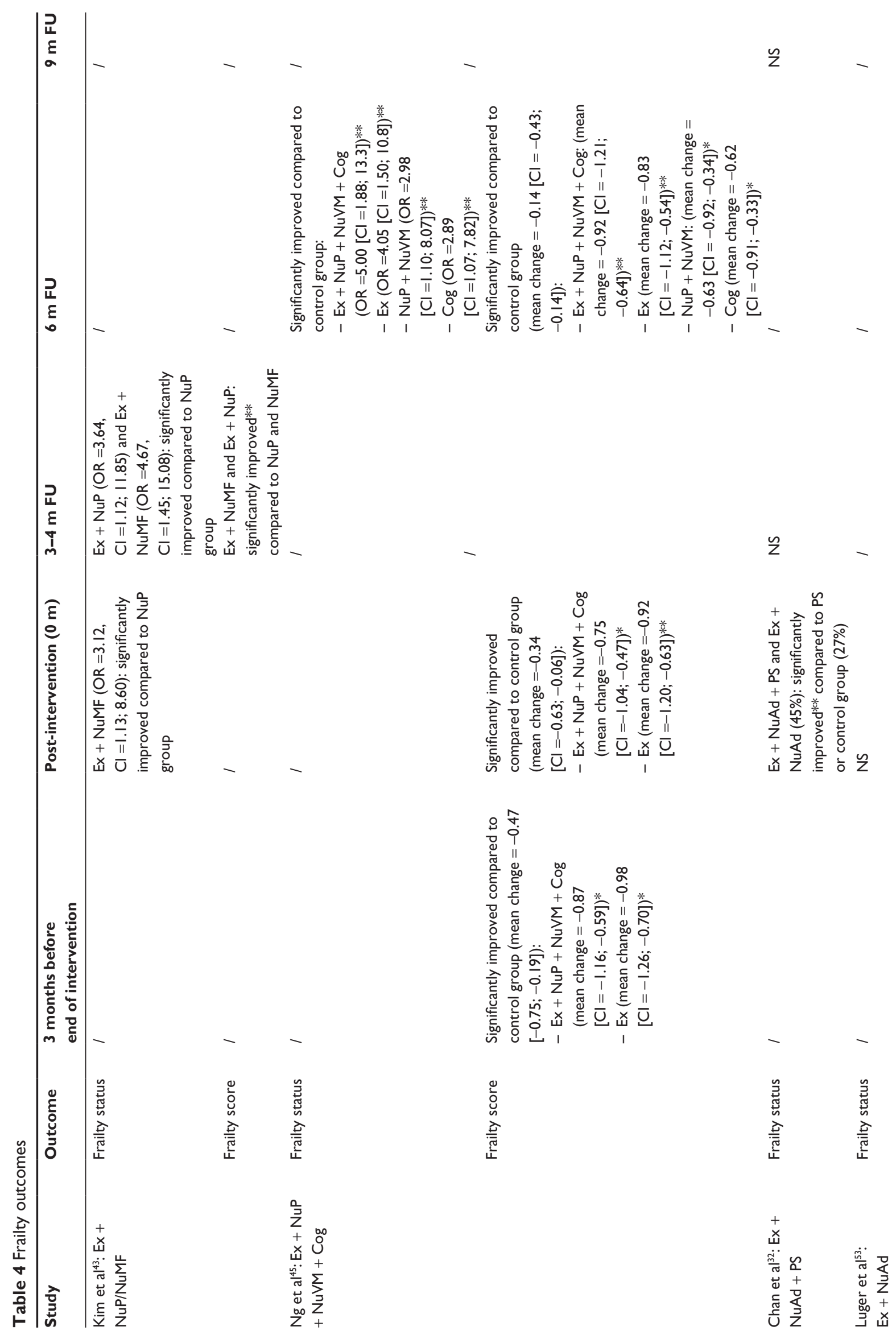




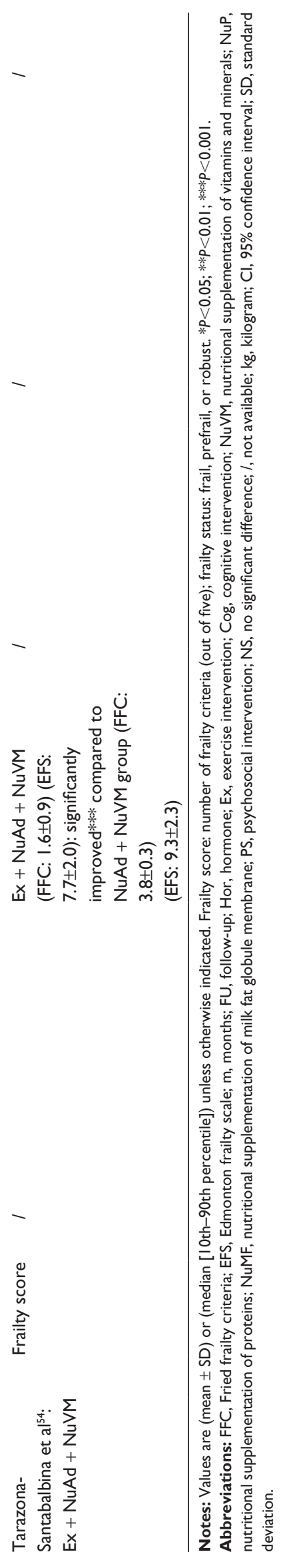

interventions including exercise. ${ }^{33,43} \mathrm{Kim}$ et al found that adding an exercise intervention to NuMF supplementation improved gait speed. ${ }^{43}$ Chin A Paw et al found significant improvements on gait speed by an exercise intervention with protein, vitamin, and mineral supplementation intervention compared to protein, vitamin, and mineral supplementation. ${ }^{33}$ Adding nutritional advice or protein supplementation to an exercise intervention showed no significant effect on gait speed, compared to exercise intervention alone. ${ }^{44,46,50}$ Also, adding a hormone intervention to a vitamin and mineral supplementation and exercise intervention showed no significant effect for gait speed. ${ }^{42}$ No significant between-group effects were found post-intervention by $\mathrm{Ex}+\mathrm{Cog}+\mathrm{NuVM}+\mathrm{NuP}$ intervention compared to single-domain interventions. ${ }^{45}$

\section{Physical activity level (low physical activity)}

Five studies examined the effect of the intervention on physical activity level (Table 7B). Adding an exercise intervention to a nutritional advice and vitamin and mineral supplementation increased physical activity. ${ }^{54}$ Another study found significantly increased physical activity by a $\mathrm{NuP}+$ $\mathrm{NuVM}$ intervention compared to the control group postintervention and at 6 months follow-up. ${ }^{45}$ Physical activity was also increased by Ex intervention alone or Ex combined with NuAd post-intervention and at 6 months follow-up; this increase remained in the Ex group compared to NuAd or control group. ${ }^{47}$ Kenny et al and Ikeda et al found no significant effect on physical activity of adding a hormone intervention to exercise and nutritional vitamin and mineral supplementation ${ }^{42}$ and adding a protein supplementation to exercise intervention, ${ }^{55}$ respectively.

\section{Impact of the intervention strategies on the consequences of frailty Change in functional abilities}

Four studies examined the effect of multi-domain interventions on functional abilities (Table 8A). Functional abilities are described by activities of daily living (ADL) and/or instrumental activities of daily living (IADL) and/or personal activities of daily living dependency or scores. There was a significant improved effect on ADL and IADL by adding an exercise intervention to a nutritional advice and vitamin and mineral supplementation intervention. ${ }^{54}$ No significant differences were found between a PS intervention (problemsolving therapy) with or without Ex + NuAd intervention compared to no psychosocial intervention or between an $\mathrm{Ex}+\mathrm{NuAd}$ intervention with or without a PS intervention compared to no Ex + NuAd intervention, ${ }^{32}$ or between an 
Table 5 Muscle mass

\begin{tabular}{|c|c|c|c|c|c|c|}
\hline Study & $\begin{array}{l}\text { Outcome and } \\
\text { method }\end{array}$ & $\begin{array}{l}3 \text { months } \\
\text { before end of } \\
\text { intervention }\end{array}$ & Post-intervention $(0 \mathrm{~m})$ & $4 \mathrm{~m}$ FU & $6 \mathrm{~m} \mathrm{FU}$ & $9 \mathrm{~m} \mathrm{FU}$ \\
\hline Kim et $\mathrm{al}^{43}:$ Ex + NuP/NuMF & $\begin{array}{l}\text { Appendicular skeletal } \\
\text { mass (kg); DXA }\end{array}$ & l & NS & NS & l & I \\
\hline Tieland et al $\left.\right|^{50}: \mathrm{Ex}+\mathrm{NuP}+\mathrm{NuVM}$ & Lean mass $(\mathrm{kg})$; DXA & l & $\begin{array}{l}\text { Treatment } \times \text { time interactions: } \\
\text { Ex }+ \text { NuP }+ \text { NuVM: significantly } \\
\text { improved compared to Ex group for } \\
\text { appendicular*** and total muscle mass** } \\
\text { Appendicular muscle mass: Ex }+ \text { NuP }+ \\
\text { NuVM: }+4.48 \% \text {; Ex: }-1.04 \% \\
\text { Total muscle mass: Ex }+\mathrm{NuP}+ \\
\text { NuVM: }+2.75 \% \text {; Ex: }-0.66 \%\end{array}$ & 1 & l & l \\
\hline $\begin{array}{l}\text { Chin A Paw et } a^{36}: \text { Ex }+ \text { NuP }+ \\
\text { NuVM }\end{array}$ & $\begin{array}{l}\text { Lean body mass }(\mathrm{kg}) \text {; } \\
\text { DXA }\end{array}$ & l & $\begin{array}{l}\text { Ex + NuP + NuVM and Ex }(+0.5 \mathrm{~kg}): \\
\text { significantly improved* compared to } \\
\mathrm{NuP}+\mathrm{NuVM} \text { or control group }(-0 . \mathrm{l} \mathrm{kg}) \\
\text { Ex }(0.2 \pm \mathrm{I} .4 \mathrm{~kg}) \text { : change }(0.47 \%) \\
\text { significantly improved* compared to } \\
\text { control group }(-1.18 \%)(-0.5 \pm \mathrm{l} .4 \mathrm{~kg})\end{array}$ & l & l & l \\
\hline $\begin{array}{l}\text { Tarazona-Santabalbina et al }\left.\right|^{54} \text { : } \\
\text { Ex + NuAd + NuVM }\end{array}$ & $\begin{array}{l}\text { Lean mass }(\mathrm{kg}) \text {; } \\
\text { BIA }\end{array}$ & I & NS & l & l & I \\
\hline Kenny et al ${ }^{42}: \mathrm{Ex}+\mathrm{NuVM}+\mathrm{Hor}$ & $\begin{array}{l}\text { Total and regional lean } \\
\text { tissue mass (kg); DXA }\end{array}$ & l & $\begin{array}{l}\text { Appendicular skeletal muscle mass: } \\
\text { NS; Lean mass: Ex + NuVM + Hor } \\
(39.6 \pm 6.1 \mathrm{~kg}): \text { significantly improved* } \\
\text { compared to Ex + NuVM }(38.1 \pm 5.2 \mathrm{~kg})\end{array}$ & l & l & l \\
\hline Rydwik et $\mathrm{al}^{46}$ : Ex + NuAd & $\begin{array}{l}\text { FFM (kg); body weight } \\
\text { minus fat mass (sum of } \\
\text { four skin folds) }\end{array}$ & l & NS & l & NS & l \\
\hline Chan et $\mathrm{al}^{32}: \mathrm{Ex}+\mathrm{NuAd}+\mathrm{PS}$ & FFM (kg); BIA method & l & l & l & l & NS \\
\hline
\end{tabular}

Notes: Values are (mean \pm SD) or (median [10th-90th percentile]) unless otherwise indicated. $* P<0.05 ; * * P<0.01 ; * * * P<0.001$.

Abbreviations: DXA, dual X-ray absorptiometry; BIA, bioelectrical impedance analysis; FFM, fat-free mass; m, months; FU, follow-up; Hor, hormone; Ex, exercise intervention; Cog, cognitive intervention; NuVM, nutritional supplementation of vitamins and minerals; NuP, nutritional supplementation of proteins; NuMF, nutritional supplementation of MFGM; PS, psychosocial intervention; NS, no significant difference; /, not available; kg, kilogram; Cl, $95 \%$ confidence interval; SD, standard deviation.

exercise intervention with protein, vitamin, and mineral supplementation intervention compared to protein, vitamin, and mineral supplementation or between a combined exercise and nutritional intervention compared to a single exercise or no intervention ${ }^{33}$ or between $\mathrm{Ex}+\mathrm{NuP}+\mathrm{NuVM}+\mathrm{Cog}$ compared to Ex or $\mathrm{NuP}+\mathrm{NuVM}$ or $\mathrm{Cog}$. ${ }^{45}$

\section{Change in physical functioning}

Three studies examined physical functioning by the short physical performance test (SPPB) (Table 8B). ${ }^{42,50,54}$ Moreover, Tarazona-Santabalbina et al also assessed the physical performance test and Tinetti balance and gait score. ${ }^{54}$ The study of Chin A Paw et al assessed performance score and fitness score. ${ }^{33}$ There was a significant beneficial effect on physical functioning of adding a hormone intervention to a vitamin and mineral supplementation and exercise intervention, ${ }^{42}$ adding an exercise intervention to a nutritional advice and vitamin and mineral supplementation intervention, ${ }^{54}$ and of an exercise intervention with protein, vitamin, and mineral supplementation intervention compared to protein, vitamin, and mineral supplementation on performance score but not fitness score. ${ }^{33}$ Adding a protein, vitamin, and mineral supplementation to an exercise intervention did not show improvements. ${ }^{50}$

Other frequently described outcomes including cognitive function, muscle power, gait ability, balance, functional lower extremity strength, and falls are described in detail in Tables 9-11.

Table S2 summarizes the secondary outcomes quality of life, social involvement, psychosocial well-being/depression, and subjective health.

\section{Discussion}

\section{Summary of evidence}

Mono-domain nonpharmacological interventions such as physical exercise have shown beneficial effects for frail elderly on gait speed, physical functioning, ${ }^{17}$ mobility, falls, functional abilities, muscle strength, body composition, 
Table 6 Muscle strength

\begin{tabular}{|c|c|c|c|c|c|}
\hline Study & Strength & $\begin{array}{l}3 \text { months } \\
\text { before end of } \\
\text { intervention }\end{array}$ & Post-intervention $(0 \mathrm{~m})$ & $4 \mathrm{~m} \mathrm{FU}$ & $6 \mathrm{~m} \mathrm{FU}$ \\
\hline Kim et $\mathrm{al}^{43}$ : & Upper & 1 & NS & NS & 1 \\
\hline $\mathrm{Ex}+\mathrm{NuP} / \mathrm{NuMF}$ & Lower & I & NS & NS & I \\
\hline Tieland et $\left.a\right|^{50}$ : & Upper & I & NS & I & I \\
\hline$E x+N u P+N u V M$ & Lower & I & NS & l & I \\
\hline Ikeda et al $\left.\right|^{55}$ : & Upper & l & NS for rowing & l & l \\
\hline \multirow[t]{2}{*}{$\mathrm{Ex}+\mathrm{NuP}$} & Lower & I & $\begin{array}{l}\text { Ex }+ \text { NuP: significantly improved leg press } \\
\text { rate* }(13.9 \% \pm 36.0 \%) \text { and knee extension } \\
\text { rate** }(9.5 \% \pm 26.3 \%) \text { compared to Ex: leg press } \\
\text { rate }(2.7 \% \pm \mid 2.5 \%) \text { and knee extension rate } \\
(-0.8 \% \pm \pm 18.2 \%)\end{array}$ & l & I \\
\hline & & & NS for hip abduction & l & I \\
\hline Chin A Paw et $a^{33}$ : & Upper & I & NS & l & I \\
\hline $\mathrm{Ex}+\mathrm{NuP}+\mathrm{NuVM}$ & Lower & I & NS & I & I \\
\hline \multirow[t]{2}{*}{$\begin{array}{l}\text { Rydwik et } \mathrm{al}^{46}: \\
\text { Ex + NuAd }\end{array}$} & Upper & I & $\begin{array}{l}\mathrm{Ex}+\mathrm{NuAd} \text { (mean change }=1.7 \mathrm{~kg}[\mathrm{Cl}=0.04 ; 3.4] \text { ) } \\
\text { and } \mathrm{Ex}(\text { mean change }=1.8 \mathrm{~kg}[\mathrm{Cl}=0.8 ; 2.8]) \text { : change } \\
\text { significantly improved }{ }^{* *} \text { compared to control group } \\
\text { (mean change }=-1.1 \mathrm{~kg}[\mathrm{Cl}=-3.2 ; 0.9] \text { ) in elbow but }\end{array}$ & 1 & NS \\
\hline & Lower & I & $\begin{array}{l}\text { NS in shoulder } \\
\text { Ex }+ \text { NuAd (mean change }=9 \mathrm{~kg}[\mathrm{Cl}=1.8 ; 16.2] \text { ) and } \\
\text { Ex (mean change }=11.9 \mathrm{~kg}[\mathrm{Cl}=6.3 ; 17.5]) \text { : change } \\
\text { significantly improved** compared to NuAd (mean } \\
\text { change }=-2.4 \mathrm{~kg}[\mathrm{Cl}=-7.9 ; 3.2] \text { ) }\end{array}$ & l & NS \\
\hline $\begin{array}{l}\text { Kwon et al }{ }^{44} \text { : } \\
\text { Ex + NuAd }\end{array}$ & Upper & I & $\begin{array}{l}\text { Ex }+ \text { NuAd: NS } \\
\text { Ex }(2.3 \pm 3.1 \mathrm{~kg}) \text { significantly improved** compared to } \\
\text { control group }(0.4 \pm 2.6 \mathrm{~kg})\end{array}$ & l & $\begin{array}{l}\text { Ex: NS } \\
\text { Ex + NuAd }(-2.1 \pm 5.0 \mathrm{~kg}) \\
\text { significantly declined** } \\
\text { compared to control group } \\
(0.1 \pm 2.4 \mathrm{~kg})\end{array}$ \\
\hline $\begin{array}{l}\mathrm{Ng} \text { et al }{ }^{45}: \mathrm{Ex}+ \\
\mathrm{NuP}+\mathrm{NuVM}+\mathrm{Cog}\end{array}$ & Lower & NS & $\begin{array}{l}\text { Significantly improved change compared to control } \\
\text { group }(\text { mean change }=0.02 \mathrm{~kg}[\mathrm{Cl}=-\mathrm{I} .08 ; \mathrm{I} . \mathrm{I}]) \\
-\mathrm{Cog} * * \text { (mean change }=2.18 \mathrm{~kg}[\mathrm{Cl}=1.08 ; 3.27]) \\
-\mathrm{Ex} * *(\text { mean change }=2.75 \mathrm{~kg}[\mathrm{Cl}=\mathrm{I} .66 ; 3.83]) \\
-\mathrm{Ex}+\mathrm{NuP}+\mathrm{NuVM}+\mathrm{Cog} * *(\text { mean change }=2.67 \\
\quad \mathrm{kg}[\mathrm{Cl}=1.58 ; 3.76])\end{array}$ & I & $\begin{array}{l}\text { Significantly improved change } \\
\text { compared to control group } \\
\text { (mean change }=-0.24 \mathrm{~kg} \\
[\mathrm{Cl}=-\mathrm{I} .34 ; 0.87]) \\
-\mathrm{Cog} * * \text { (mean change }=1.98 \mathrm{~kg} \\
\quad[\mathrm{Cl}=0.87 ; 3.09]) \\
-\mathrm{Ex} \text { (mean change }=1.41 \mathrm{~kg} \\
\quad[\mathrm{Cl}=0.3 \mathrm{I} ; 2.5 \mathrm{I}]) \\
-\mathrm{Ex}+\mathrm{NuP}+\mathrm{NuVM}+\mathrm{Cog}^{* *} \\
\quad(\text { mean change }=2.35 \mathrm{~kg} \\
\quad[\mathrm{Cl}=\mathrm{I} .25 ; 3.44])\end{array}$ \\
\hline Kenny et $\mathrm{a}^{42}$ : Ex + & Upper & I & NS & I & I \\
\hline NuVM + Hor & Lower & l & $\begin{array}{l}\text { Ex + NuVM + Hor }(484 \pm I 47 N) \text { : significantly } \\
\text { improved* compared to Ex + NuVM }(447 \pm I 28 N)\end{array}$ & 1 & I \\
\hline $\begin{array}{l}\text { Hennessey et } \mathrm{al}^{41} \text { : } \\
\text { Ex }+ \text { Hor }\end{array}$ & Lower & I & $\begin{array}{l}\text { Hor** }(P=0.007) \text { and } E x^{* * *}(P<0.0005) \text { significantly } \\
\text { improved strength compared to control group }\end{array}$ & I & I \\
\hline
\end{tabular}

Notes: Values are (mean $\pm \mathrm{SD}$ ) or (median [10th-90th percentile]) unless otherwise indicated. $* P<0.05 ; * * P<0.01 ; * * * P<0.001$.

Abbreviations: DXA, dual X-ray absorptiometry; BIA, bioelectrical impedance analysis; FFM, fat-free mass; m, months; FU, follow-up; Hor, hormone; Ex, exercise intervention; Cog, cognitive intervention; NuVM, nutritional supplementation of vitamins and minerals; NuP, nutritional supplementation of proteins; NuMF, nutritional supplementation of MFGM; PS, psychosocial intervention; NS, no significant difference; /, not available; kg, kilogram; Cl, 95\% confidence interval; SD, standard deviation.

and frailty. ${ }^{56}$ However, no systematic reviews exist on the effectiveness of multi-domain interventions, nor the contribution of a mono-domain intervention to a multi-domain intervention. This systematic review included twelve studies investigating the effect of a multi-domain intervention in frail elderly on frailty status and score, cognition, muscle mass strength and power, and functional, emotional, and social outcomes. These studies were heterogeneous in terms of included participants (frailty diagnostic tool), intervention strategies (type of interventions, number of interventions, 
Table 7 Gait speed and physical activity

\begin{tabular}{|c|c|c|c|c|c|}
\hline Study & $\begin{array}{l}3 \text { months } \\
\text { before end of } \\
\text { intervention }\end{array}$ & Post-intervention $(0 \mathrm{~m})$ & 3-4 m FU & $6 \mathrm{~m} \mathrm{FU}$ & $9 \mathrm{~m}$ FU \\
\hline \multicolumn{6}{|l|}{ A: Gait speed $(\mathrm{m} / \mathrm{s})$} \\
\hline Kim et $a^{133-}:$ Ex + NuP/NuMF & & $\begin{array}{l}\text { Ex }+ \text { NuMF }(\% \text { change }=14.7 \pm 4.1)(\mathrm{Cl}=6.4 ; 23.1) \text { : } \\
\text { change significantly improved* compared to } \\
\text { NuMF }(\% \text { change }=2.1 \pm 1.9)(\mathrm{Cl}=-1.8 ; 5.9) \text { or NuP } \\
(\% \text { change }=3.6 \pm 2.7)(\mathrm{Cl}=-1.9 ; 9.1)\end{array}$ & NS & I & I \\
\hline $\begin{array}{l}\text { Tieland et a }\left.\right|^{50}: \text { Ex + NuP + } \\
\text { NuVM }\end{array}$ & l & NS & l & I & I \\
\hline Rydwik et al ${ }^{46}: \mathrm{Ex}+\mathrm{NuAd}$ & 1 & NS & 1 & NS & I \\
\hline Kwon et $\mathrm{al}^{44}: \mathrm{Ex}+\mathrm{NuAd}$ & 1 & NS & 1 & NS & I \\
\hline $\begin{array}{l}\text { Chin A Paw et al3: Ex }+ \\
\text { NuP + NuVM }\end{array}$ & 1 & $\begin{array}{l}\text { Ex }+ \text { NuP }+ \text { NuVM or Ex }(0.06 \pm 0.1): \text { significantly } \\
\text { improved** compared to NuP }+ \text { NuVM or control } \\
\text { group }(0.0 \pm 0.04)\end{array}$ & l & I & I \\
\hline $\begin{array}{l}\text { Kenny et al }{ }^{42}: \text { Ex }+ \\
\text { NuVM + Hor }\end{array}$ & l & NS & l & I & I \\
\hline $\begin{array}{l}\mathrm{Ng} \text { et al } \mathrm{a}^{45}: \mathrm{Ex}+\mathrm{NuP}+ \\
\mathrm{NuVM}+\mathrm{Cog}\end{array}$ & NS & NS & I & NS & I \\
\hline \multicolumn{6}{|l|}{ B: Physical activity } \\
\hline $\begin{array}{l}\text { Kenny et a } \mathbf{l}^{42}: \text { Ex }+ \\
\text { NuVM + Hor }\end{array}$ & 1 & NS & 1 & I & I \\
\hline Rydwik et al ${ }^{47}:$ Ex + NuAd & 1 & $\begin{array}{l}\text { Ex and Ex + NuAd: change significantly improved* } \\
\text { compared to control group }\end{array}$ & l & $\begin{array}{l}\text { Ex: change significantly } \\
\text { improved* compared to } \\
\text { control group or NuAd }\end{array}$ & I \\
\hline $\begin{array}{l}\mathrm{Ng} \text { et al }{ }^{45}: \mathrm{Ex}+\mathrm{NuP}+ \\
\mathrm{NuVM}+\mathrm{Cog}\end{array}$ & NS & $\begin{array}{l}\mathrm{NuP}+\mathrm{NuVM} \text { (mean change }=96.2[\mathrm{Cl}=57.8 \\
\text { I34.7]): change significantly improved } \\
\text { to control group (mean change }=20.5[\mathrm{Cl}=-17.0 ; \\
58 . \mathrm{I}] \text { ) }\end{array}$ & 1 & $\begin{array}{l}\mathrm{NuP}+\mathrm{NuVM} \\
\text { (mean change }=1 \mathrm{I} 0.1 \\
[\mathrm{Cl}=7 \mathrm{I} .9 ; 148.2]): \\
\text { significantly improved** } \\
\text { compared to control } \\
\text { group (mean change }=34.8 \\
[\mathrm{Cl}=-2.99 ; 72.6])\end{array}$ & I \\
\hline $\begin{array}{l}\text { Tarazona-Santabalbina } \\
\text { et al }{ }^{54}: \text { Ex + NuAd + NuVM }\end{array}$ & I & 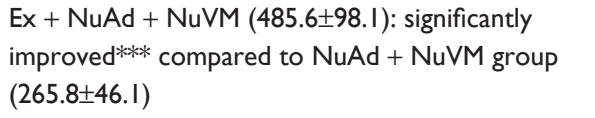 & l & I & I \\
\hline Ikeda et al ${ }^{55}: \mathrm{Ex}+\mathrm{NuP}$ & I & NS & I & I & I \\
\hline
\end{tabular}

Notes: Values are (mean $\pm S D$ ) or (median [10th-90th percentile]) unless otherwise indicated. $* P<0.05 ; * * P<0.01 ; * * * P<0.001$.

Abbreviations: $m$, months; FU, follow-up; Hor, hormone; Ex, exercise intervention; Cog, cognitive intervention; NuVM, nutritional supplementation of vitamins and minerals; NuP, nutritional supplementation of proteins; NuMF, nutritional supplementation of milk fat globule membrane; PS, psychosocial intervention; NS, no significant difference; /, not available; kg, kilogram; Cl, 95\% confidence interval; SD, standard deviation.

and combinations of interventions), and intervention duration. Overall, multi-domain interventions show a greater beneficial impact compared to mono-domain interventions (eg, nutritional intervention alone) or usual care for frailty characteristics, physical functioning, and muscle mass and strength. To be more specific, physical exercise seems to play an essential role in the multi-domain intervention, with some improvements by an additional intervention (eg, nutritional intervention). As suggested in previous reviews, the positive effects of nutritional supplementation increase when associated with physical exercise and the positive effects of physical exercise increase when associated with nutritional supplementation..$^{22,57-59}$ Also, it could be claimed that the physical exercise component accounts for the greatest improvements, which has also been suggested in reviews discussing frail ${ }^{28}$ and sarcopenic elderly. ${ }^{22}$

\section{Multi-domain interventions improve frailty characteristics and physical functioning more effectively than mono-domain interventions}

Overall, this review indicates that multi-domain interventions are more effective than mono-domain interventions for several outcomes, such as frailty status or score. More specifically, the combination of physical exercise and nutritional intervention yielded a more positive result on frailty 
Table 8 Functional abilities and physical functioning

\begin{tabular}{|c|c|c|c|c|c|}
\hline$\overline{\text { Study }}$ & $\begin{array}{l}3 \text { months } \\
\text { before end of } \\
\text { intervention }\end{array}$ & Post-intervention $(0 \mathrm{~m})$ & 3-4 m FU & $6 \mathrm{~m} \mathrm{FU}$ & $9 \mathrm{~m} \mathrm{FU}$ \\
\hline \multicolumn{6}{|c|}{ A: Functional abilities (ADL/IADL/PADL) } \\
\hline $\mathrm{Ng}$ et $\mathrm{al}^{45}: \mathrm{Ex}+\mathrm{NuP}+\mathrm{NuVM}+\mathrm{Cog}$ & NS & NS & l & NS & l \\
\hline & NS & NS & l & NS & l \\
\hline Chan et $\mathrm{a}^{32}: \mathrm{Ex}+\mathrm{NuAd}+\mathrm{PS}$ & l & NS & NS & l & NS \\
\hline $\begin{array}{l}\text { Chin A Paw et a }{ }^{33}: \text { Ex }+\mathrm{NuP}+ \\
\text { NuVM }\end{array}$ & l & NS & I & I & l \\
\hline $\begin{array}{l}\text { Tarazona-Santabalbina et } \mathrm{al}^{54} \text { : } \\
\text { Ex + NuAd + NuVM }\end{array}$ & l & 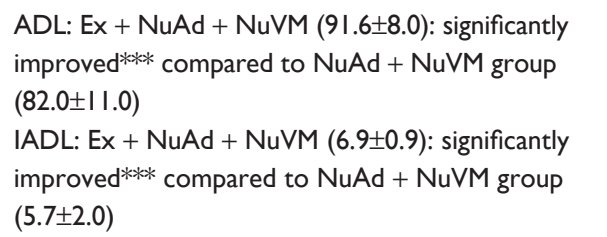 & l & I & l \\
\hline \multicolumn{6}{|l|}{ B: Physical functioning } \\
\hline $\begin{array}{l}\text { SPPB } \\
\text { Tieland et al }\left.\right|^{50}: \mathrm{Ex}+\mathrm{NuP}+\mathrm{NuVM}\end{array}$ & l & NS & l & l & l \\
\hline $\begin{array}{l}\text { SPPB } \\
\text { Kenny et al }{ }^{42}: \mathrm{Ex}+\mathrm{NuVM}+\mathrm{Hor}\end{array}$ & I & $\begin{array}{l}\text { Ex + NuVM + Hor }(10.7 \pm 1.9): \text { significantly } \\
\text { improved* compared to Ex + NuVM }(10.1 \pm 1.8)\end{array}$ & l & l & I \\
\hline $\begin{array}{l}\text { SPPB } \\
\text { Tarazona-Santabalbina et } \mathrm{al}^{54} \text { : } \\
\text { Ex + NuAd + NuVM }\end{array}$ & l & $\begin{array}{l}\text { Ex + NuAd + NuVM }(9.5 \pm 1.8): \text { significantly } \\
\text { improved** compared to NuAd + NuVM group } \\
(7.1 \pm 2.8)\end{array}$ & l & I & l \\
\hline $\begin{array}{l}\text { PPT } \\
\text { Tarazona-Santabalbina et al }{ }^{54} \text { : } \\
\text { Ex + NuAd + NuVM }\end{array}$ & l & $\begin{array}{l}\text { Ex + NuAd + NuVM (23.5 } \pm 5.9): \text { significantly } \\
\text { improved*** compared to NuAd + NuVM group } \\
(16.5 \pm 5.1)\end{array}$ & l & 1 & I \\
\hline $\begin{array}{l}\text { Tinetti } \\
\text { Tarazona-Santabalbina et } a^{54} \\
\text { Ex + NuAd + NuVM }\end{array}$ & 1 & $\begin{array}{l}\text { Ex }+ \text { NuAd + NuVM }(24.5 \pm 4.4): \text { significantly } \\
\text { improved** compared to NuAd + NuVM group } \\
(21.7 \pm 4.5)\end{array}$ & 1 & I & 1 \\
\hline $\begin{array}{l}\text { Performance score } \\
\text { Chin A Paw et a }{ }^{33}: \mathrm{Ex}+\mathrm{NuP}+ \\
\text { NuVM }\end{array}$ & I & $\begin{array}{l}\text { Ex and Ex }+\mathrm{NuP}+\mathrm{NuVM} \text { significantly improved } * * * \\
\text { compared to NuP }+ \text { NuVM or control group }\end{array}$ & 1 & 1 & 1 \\
\hline $\begin{array}{l}\text { Fitness score } \\
\text { Chin A Paw et a }{ }^{33}: \mathrm{Ex}+\mathrm{NuP}+ \\
\mathrm{NuVM}^{33}\end{array}$ & I & $\begin{array}{l}\text { NS: Ex and Ex + NuP NuVM NS different } \\
\text { compared to NuP + NuVM or control group }\end{array}$ & 1 & I & 1 \\
\hline
\end{tabular}

Notes: Values are (mean $\pm \mathrm{SD}$ ) or (median [10th-90th percentile]) unless otherwise indicated. $* P<0.05 ; * * P<0.01 ; * * * P<0.001$

Abbreviations: FU, follow-up; TUG, timed up and go test; Hor, hormone; Ex, exercise intervention; Cog, cognitive intervention; NuVM, nutritional supplementation of vitamins and minerals; NuP, nutritional supplementation of proteins; PS, psychosocial intervention; NS, no significant difference; /, not available; s, seconds; CI, $95 \%$ confidence interval; SD, standard deviation.

status or score compared to a nutritional intervention ${ }^{43,45,54}$ or a physical exercise intervention. ${ }^{45}$ This effect was not found consistently and probably partly depends on variables such as the type and frequency of intervention and target group.

The impact of physical exercise on frailty characteristics was previously described. ${ }^{29}$ It is now of particular interest to mark the added value of the combination of an exercise and nutritional intervention, underlining the contribution of the nutritional intervention to frailty improvements. Moreover, this positive effect on frailty seems to be more prolonged

Table 9 Muscle power

\begin{tabular}{|c|c|c|c|}
\hline Study & $\begin{array}{l}\text { Post-intervention } \\
(0 \mathrm{~m})\end{array}$ & $3 \mathrm{~m}$ FU & $9 \mathrm{~m}$ FU \\
\hline Chan et $\mathrm{al}^{32}: \mathrm{Ex}+\mathrm{NuAd}+\mathrm{PS}$ & NS & $\begin{array}{l}\text { Ex + NuAD + PS and PS }(2.7 \mathrm{I} \pm 6.08 \mathrm{~kg}): \\
\text { significantly improved* change compared } \\
\text { to Ex + NuAd or control group } \\
(0.18 \pm 6.68 \mathrm{~kg})\end{array}$ & $\begin{array}{l}\text { Ex + NuAD + PS and PS }(-3.52 \pm 9.65 \mathrm{~kg}): \\
\text { significantly improved* change compared to } \\
\text { Ex + NuAd or control group }(-7.14 \pm 8.74 \mathrm{~kg})\end{array}$ \\
\hline Kenny et $\mathrm{al}^{42}:$ Ex $+\mathrm{NuVM}+$ Hor & NS & i & l \\
\hline
\end{tabular}

Notes: Values are (mean $\pm \mathrm{SD}$ ) or (median [10th-90th percentile]) unless otherwise indicated. $* \mathrm{P}<0.05$.

Abbreviations: m, months; FU, follow-up; Hor, hormone; Ex, exercise intervention; NuVM, nutritional supplementation of vitamins and minerals; PS, psychosocial intervention; NS, no significant difference; /, not available; kg, kilogram; SD, standard deviation. 
Table 10 Gait ability, balance, functional lower extremity strength, falls

\begin{tabular}{|c|c|c|c|c|c|}
\hline Study & $\begin{array}{l}3 \text { months } \\
\text { before end of } \\
\text { intervention }\end{array}$ & Post-intervention $(0 \mathrm{~m})$ & 3-4 m FU & $6 \mathrm{~m} \mathrm{FU}$ & $9 \mathrm{~m} \mathrm{FU}$ \\
\hline \multicolumn{6}{|l|}{ A: Gait ability (TUG) } \\
\hline Kim et al ${ }^{43}:$ Ex + NuP/NuMF & l & $\begin{array}{l}\text { Ex }+ \text { NuMF }(\% \text { change }=-14.4 \pm 2.0) \\
(\mathrm{Cl}=-13.8 ;-9.9) \text { and } \mathrm{Ex}+\mathrm{NuP} \\
(\% \text { change }=-18.5 \pm 2.1)(\mathrm{Cl}=-22.9 ; \\
-14.0) \text { : change significantly improved } * * * \\
\text { compared to NuMF }(\% \text { change } \\
=-6.1 \pm 2.6)(\mathrm{Cl}=-1 \mathrm{I} .6 ;-0.7) \text { or NuP }(\% \\
\text { change }=-3.0 \pm 2.6)(\mathrm{Cl}=-8.3 ; 2.3)\end{array}$ & NS & l & l \\
\hline Rydwik et al ${ }^{46}: \mathrm{Ex}+\mathrm{NuAd}$ & l & NS & l & NS & l \\
\hline Kenny et $\mathrm{a}^{42}: \mathrm{Ex}+\mathrm{NuVM}+\mathrm{Hor}$ & l & NS & l & l & I \\
\hline Ikeda et $a^{\mid 55}: \mathrm{Ex}+\mathrm{NuP}$ & l & NS & 1 & l & l \\
\hline \multicolumn{6}{|l|}{ B: Balance } \\
\hline \multicolumn{6}{|l|}{ Dynamic balance } \\
\hline \multirow[t]{2}{*}{ Rydwik et a ${ }^{46}:$ Ex + NuAd } & l & NS (modified figure eight) & l & NS & l \\
\hline & l & $\begin{array}{l}\text { Ex }+\mathrm{NuAd} \text { (mean change }=-\mathrm{I} . \mathrm{I}[\mathrm{Cl} \\
=-3.2 ; \mathrm{I}]) \text { : score step test significantly } \\
\text { decreased* compared to Ex (mean } \\
\text { change }=3.2[\mathrm{Cl}=0.9 ; 5.5])\end{array}$ & 1 & NS & I \\
\hline Ikeda et $\mathrm{al}^{55}: \mathrm{Ex}+\mathrm{NuP}$ & l & $\begin{array}{l}\text { Ex + NuP: functional reach test } \\
\text { improvement rate significantly } \\
\text { improved* }(11.0 \% \pm 22.0 \%) \text { compared to } \\
\text { Ex }(1.0 \% \pm 17.0 \%)\end{array}$ & 1 & l & I \\
\hline \multicolumn{6}{|l|}{ Static balance } \\
\hline Chan et $a^{32}:$ Ex + NuAd + PS & l & NS (single leg stance) & NS & l & NS \\
\hline Kenny et $\mathrm{a}^{42}: \mathrm{Ex}+\mathrm{Hor}+\mathrm{NuVM}$ & l & NS (singe leg stance) & 1 & l & I \\
\hline \multirow[t]{2}{*}{ Rydwik et al ${ }^{46}:$ Ex + NuAd } & l & NS (tandem stance) & 1 & NS & l \\
\hline & l & NS (single leg stance) & l & NS & l \\
\hline Kwon et al ${ }^{44}: \mathrm{Ex}+\mathrm{NuAd}$ & l & NS (stork stance) & l & NS & l \\
\hline \multirow[t]{2}{*}{ Chin A Paw et $\mathrm{al}^{33}: \mathrm{Ex}+\mathrm{NuP}+\mathrm{NuVM}$} & l & NS (tandem stance) & l & l & l \\
\hline & l & $\begin{array}{l}\text { Ex }+ \text { NuP }+ \text { NuVM and Ex }(4[-7 ; 17]) \text { : } \\
\text { change in score for balancing on balance } \\
\text { board significantly improved* compared } \\
\text { to NuP + NuVM and control group: }(2 \\
[-12 ; 13])\end{array}$ & l & l & l \\
\hline \multicolumn{6}{|l|}{ Dynamic and static balance } \\
\hline \multicolumn{6}{|l|}{$+\mathrm{NuVM}$} \\
\hline \multicolumn{6}{|l|}{ C: Functional lower extremity strength } \\
\hline Rydwik et al ${ }^{46}:$ Ex + NuAd & l & NS & l & NS & I \\
\hline Chin A Paw et $a^{33}: \mathrm{Ex}+\mathrm{NuP}+\mathrm{NuVM}$ & l & $\begin{array}{l}\text { Ex + NuP + NuVM and Ex }(-2.3 \mathrm{~s} \\
\text { for chair stand }[-7.7 ; 1.4]): \text { change } \\
\text { significantly improved* compared to NuP } \\
+\mathrm{NuVM} \text { and control group }(-1.0 \mathrm{~s} \text { for } \\
\text { chair stand }[-6.4 ; 3.8])\end{array}$ & l & l & I \\
\hline Kenny et $\mathrm{a}^{42}: \mathrm{Ex}+\mathrm{Hor}+\mathrm{NuVM}$ & l & NS & l & l & l \\
\hline Tieland et $\mathrm{al}^{50}: \mathrm{Ex}+\mathrm{NuP}+\mathrm{NuVM}$ & l & NS & l & l & l \\
\hline \multicolumn{6}{|l|}{ D: Falls } \\
\hline Ng et al ${ }^{45}: \mathrm{Ex}+\mathrm{NuP}+\mathrm{NuVM}+\mathrm{Cog}$ & NS & NS & 1 & NS & 1 \\
\hline $\begin{array}{l}\text { Tarazona-Santabalbina et al } l^{54}: \text { Ex + NuAd } \\
+ \text { NuVM }\end{array}$ & l & NS & 1 & l & 1 \\
\hline
\end{tabular}

Notes: Values are (mean $\pm S D$ ) or (median [10th-90th percentile]) unless otherwise indicated. $* P<0.05 ; * * * P<0.001$.

Abbreviations: FU, follow-up; SPPB, short physical performance test; PPT, physical performance test; Hor, hormone; Ex, exercise intervention; Cog, cognitive intervention; NuVM, nutritional supplementation of vitamins and minerals; NuP, nutritional supplementation of proteins; NuMF, nutritional supplementation of milk fat globule membrane; PS, psychosocial intervention; NS, no significant difference; /, not available; s, seconds; Cl, 95\% confidence interval; SD, standard deviation. 
Table I I Cognitive status

\begin{tabular}{ll}
\hline Study & Post-intervention $\mathbf{( 0 ~} \mathbf{m})$ \\
\hline van de Rest ${ }^{5}:$ Ex + NuP + NuVM & Episodic memory: NS \\
& Attention and working memory: NS \\
& Information processing speed* Ex + NuP + NuVM $(0.08 \pm 0.5 \mathrm{I})$ : change significantly improved compared to \\
& NuP + NuVM $(-0.23 \pm 0.19)$ \\
& Executive functioning: NS
\end{tabular}

Notes: Values are (mean $\pm S D$ ) or (median [10th-90th percentile]) unless otherwise indicated. $* P<0.05$.

Abbreviations: Ex, exercise intervention; NuVM, nutritional supplementation of vitamins and minerals; NuP, nutritional supplementation of proteins; NS, no significant difference; SD, standard deviation.

in multi-domain compared to mono-domain interventions. These observations underpin the inherent characteristics of the frailty syndrome: a system-wide syndrome that demands a system-wide approach.

Besides frailty status and score, multi-domain interventions also improved physical functioning (eg, SPPB) more effectively compared to a nutritional intervention, ${ }^{33,54}$ a combined exercise and nutritional intervention ${ }^{42}$ or no intervention. ${ }^{33}$ This is plausible as multiple interventions can act on multiple levels of physical functioning and therefore affect the score of a multifaceted test. Although there is a tendency for improved results by multi-domain interventions, particularly the combination of an exercise and nutritional intervention, ${ }^{33,43,46}$ the effects were less conclusive for the individual components of the physical functioning test (gait speed, gait ability, balance, and functional lower extremity strength).

Muscle mass and strength showed a tendency to be improved more effectively by multi-domain compared to mono-domain interventions. These results were previously described in reviews in sarcopenic populations. ${ }^{22,57}$ More specifically, the combined physical exercise and nutritional intervention showed a tendency to improve muscle mass and muscle strength more than exercise or nutritional intervention alone. Skeletal muscle strength does not solely depend on muscle mass but is a function of multiple factors such as nutritional, hormonal, and neurological components and physical activity. ${ }^{60,61}$ Therefore, it is plausible that the combination of two or more of these interventions will add to the intervention efficacy. In addition, the results seemed to be more consistent when the intervention duration was at least 4 months. ${ }^{33,42,50}$

The beneficial effects of an exercise intervention alone on frailty ${ }^{62}$ muscle outcomes ${ }^{63}$ physical functioning, ${ }^{17}$ quality of life, ${ }^{64}$ depression, ${ }^{65}$ and cognition ${ }^{66}$ have been described extensively. Although this review did not focus on singledomain interventions, it was observed that the exercise intervention on its own consistently contributed to the core effects on frailty, muscle mass, and muscle strength. ${ }^{33,44} 46$ Therefore, the role of the exercise intervention seems primordial as part of a multi-domain intervention. Exercise program characteristics (frequency, intensity, duration, and type of training) influence its effects and must therefore be optimized. According to the recent literature, an optimal exercise intervention for frail elderly is performed at least three times a week with progressive moderate intensity for 30-45 minutes per session and for a duration of at least 5 months. The optimal type of exercise intervention is a multicomponent intervention covering aerobic exercise, strength training, balance, and flexibility ${ }^{67,68}$ but depends on the outcome that must be improved. The content of the exercise interventions in the different studies in this review is diverse, including several interventions with insufficient training stimulus, for example, training only once a week. ${ }^{44}$ Exercise interventions with a clear insufficient dose or intensity of training cannot be expected to have an effect, for example, on muscle strength.

The additional effect of combining physical exercise with a nutritional intervention is frequently observed, however not consistently. One argument could be that due to the energy and protein deficits as a consequence of the malnutrition of the participants, the effect of the nutritional intervention is suboptimal because the nutrients are first used to resolve these energy and protein deficits. Malnutrition is often present in community-dwelling elderly; ${ }^{69}$ moreover, frail elderly have lower intakes of energy, protein, and/or several micronutrients compared to non-frail elderly. ${ }^{70,71}$ Malnutrition is a result of several factors including anabolic resistance. Anabolic resistance is an aging-associated resistance in response to the positive effects of dietary protein on protein synthesis that elderly develop. ${ }^{72}$ Several mechanisms underlie anabolic resistance such as splanchnic sequestration of amino acids, decreased postprandial availability of amino acids, and decreased muscle uptake of dietary amino acids. ${ }^{72}$ 
Protein intake combined with exercise could increase the anabolic stimulus of exercise. However, due to the anabolic resistance and to obtain beneficial effects of exercise interventions, frail elderly need larger protein intakes. Similarly, physical exercise improves muscle sensitivity to protein or amino acid uptake, consequently counteracting anabolic resistance. Furthermore, the quality and quantity of the nutritional intervention must be emphasized: with insufficient protein intake, an additional effect compared to the exercise intervention alone cannot be expected, similar as when the exercise stimulus is insufficient. Guidelines recommend an intake of 1.2-1.5 g protein/ $\mathrm{kg}$ bodyweight/day for frail elderly. In addition, each meal should contain $20-40 \mathrm{~g}$ protein in order to stimulate muscle protein synthesis in the elderly. ${ }^{73-76}$ Therefore, nutritional supplementation to reach this threshold must include an assessment of the daily protein intake of the participants. This was done in only one study included in this review. ${ }^{50}$ As a result, nutritional interventions may be inadequate and results may be misinterpreted. This could lead to an underestimation of the value of nutritional supplementation. To exclude this argument in future, it is important 1) to implement nutritional interventions tailored to the nutritional intake and habits of the participants or 2) to restore the participant's nutritional status both before the start and during the intervention.

Nutritional status can be targeted directly by adding proteins or nutrients to the diet or indirectly by advising the participant about the importance of several nutrients and how to add them to the diet. Nutritional interventions in this review were heterogeneous in terms of content ( $\mathrm{NuAd}$, NuP, NuVM, and NuMF) and design (daily, once a month), resulting in variability in effects. Therefore, no reliable conclusions regarding the stronger intervention can be drawn. To all intents and purposes, direct nutritional supplementation can be advised to achieve direct effects on nutritional status, moreover, higher protein intake was associated with less likelihood of being frail (based on Fried criteria). ${ }^{77}$ However, teaching the participant to evaluate and adapt his/her own nutritional intake will improve the sustainability of the effect and the compliance to the intervention.

In conclusion, multi-domain interventions, where both exercise and nutritional interventions are optimally designed, reveal a stronger effect as frailty, physical functioning, and muscle mass and strength depend on multiple factors. As a result, we recommend the exercise intervention as an essential part of future multi-domain intervention studies. Moreover, attention must be paid to the design of both exercise and nutritional interventions to elucidate the optimal effect of the interventions. In addition, the compliance of the participants to the interventions is of crucial importance. In turn, compliance to the exercise intervention is influenced by the supervision on and location of the intervention.

\section{Inconsistent effects on functional abilities, falls, and psychosocial outcomes}

No consistent effects of multi-domain interventions on functional abilities, falls or quality of life, psychosocial behavior, or depression were found. However, beneficial effects may have been missed due to a low number of studies examining these outcomes or insufficient power, as several studies did not do a proper sample size calculation for these outcomes or did not include these outcomes as primary outcomes. Previous studies describe improved functional abilities by an exercise intervention but underline the importance that the intervention exercises are functional and task specific (eg, exercising chair rises) in order to improve functional abilities. ${ }^{78,79}$ Furthermore, recent systematic reviews found conflicting results of the effect of physical activity on quality of life: one review described no consistent effect in elderly with mobility problems, physical disability, and/or multimorbidity, ${ }^{80}$ whereas another review described a positive and consistent association in elderly. ${ }^{81}$

\section{Methodological considerations}

Multiple studies were excluded during the study selection, mainly because the authors did not report outcomes stratified by interventions, for example, the Frailty Intervention Trial by Cameron et al. ${ }^{82}$ These studies, often reporting interdisciplinary team-based approaches, assess affected domains by a comprehensive geriatric assessment and thereafter tailor the treatment to the goals, capacity, and context of the individual. ${ }^{20}$ As authors did not discriminate between intervention combinations, the interpretability of the effectiveness of the intervention components is inherently impossible. ${ }^{83}$ The exclusion of these types of articles confines the scope of this review, which was considered reasonable given the goal of the review.

Thanks to the assistance of an expert librarian in the citation, reference and author search, and the inclusion of studies reported in four languages; this review considerably reduced the risk for selection bias. Although the study selection based on title and abstract was done by one reviewer, all relevant articles should have been retrieved as this step was thoroughly performed and in case of doubt, the full article was read and discussed with a second reviewer. 
Methodological quality of the included studies ranged from medium to high scores. This indicates that the observed effects are not likely to be overestimated. Moreover, as almost all studies performed poorly with regard to the prospective calculation of the study sample size, the likelihood of type II errors increases, meaning that some nonsignificant results may be falsely considered as nonsignificant. This problem should be addressed in future studies, improving overall study quality.

As the primary focus of this review is to determine the effect of different multi-domain interventions, effects over time within one intervention group were not covered and will not be discussed further. Recent reviews can be consulted for a literature review of these over time effects (eg, Denison et $\mathrm{al}^{22}$ ).

\section{Remaining and upcoming questions and challenges for future studies}

Several questions remain, due to the limited number of studies. First, intervention effects on cognition, social involvement, or some functional outcomes remain unclear, as well as the contribution of several mono-domain interventions (eg, hormonal intervention). Therefore, they should be a focus of future research. Second, the optimal duration of intervention to obtain the effects on frailty status or physical functioning could not be derived due to too small number of studies. Similarly, the persistence of the achieved effects is difficult to discuss as only five studies included follow-up measurements in their study. Third, more studies should examine the ability of the interventions to prevent or treat frailty, as considerable literature describes that multi-domain interventions have this potential. ${ }^{13,16}$ Essentially, researchers are encouraged to investigate their results from a broader perspective. A core outcome set for these types of studies consisting of following measures is suggested: 1) frailty status, score, and/or characteristics; 2) muscle outcomes (mass and strength); 3) physical outcomes (at least functional abilities and one physical functioning test); 4) cognition, social outcomes, and/or psychological well-being.

In addition, new questions arise. First, heterogeneous populations are considered as (pre)frail elderly as a broad spectrum of frailty screening tools is used in research and clinical practice. Not only were different frailty definitions used, also considerable variety was observed within one type of definition, challenging the generalizability of the intervention. Ultimately, the development of a well-accepted operationalized frailty screening tool will improve homogeneity in study populations and will contribute to the understanding of the results. Second, following questions arise: "What is the optimal moment to tackle frailty by an intervention (preventive or in early pre-frailty stage)?" and "How can participants be motivated to adhere to the intervention program (personal characteristics, program factors, environmental factors)?"

\section{Conclusion}

These limited but promising data highlight the potential of the physical exercise component as a standard intervention component, optimally combined with at least a nutritional intervention. Hereby, adequate design of interventions will improve results. Multi-domain interventions were found to be more effective than mono-domain interventions for improving frailty status and physical functioning. Also, a multidomain intervention tended to yield more positive outcomes for muscle mass and strength. Eventually, understanding the contribution of each mono-domain intervention would pave the way to optimize and prioritize the frailty syndrome management. Finally, diverse frailty definitions cause heterogeneous study populations and urge the development of an overall accepted operationalized frailty definition.

\section{Acknowledgments}

Marleen Michels participated in the database search. All authors agreed to publish the paper. Funding for this systematic review was provided by internal funding of the University of Leuven, KU Leuven.

\section{Author contributions}

All authors contributed toward data analysis, drafting and revising the paper and agree to be accountable for all aspects of the work.

\section{Disclosure}

The authors report no conflicts of interest in this work.

\section{References}

1. Walston J, Hadley EC, Ferrucci L, et al. Research agenda for frailty in older adults: toward a better understanding of physiology and etiology: summary from the American Geriatrics Society/National Institute on Aging Research Conference on Frailty in Older Adults. J Am Geriatr Soc. 2006;54(6):991-1001.

2. Gielen E, Verschueren S, O’Neill TW, et al. Musculoskeletal frailty: a geriatric syndrome at the core of fracture occurrence in older age. Calcif Tissue Int. 2012;91(3):161-177.

3. Bouillon K, Kivimaki M, Hamer M, et al. Measures of frailty in population-based studies: an overview. BMC Geriatr. 2013;13:64.

4. Abellan van Kan G, Rolland Y, Houles M, Gillette-Guyonnet S, Soto M, Vellas B. The assessment of frailty in older adults. Clin Geriatr Med. 2010;26(2):275-286.

5. Fried L, Tangen C, Walston J, et al. Frailty in older adults: evidence for a phenotype. J Gerontol. 2001;56A(3):M146-M156. 
6. Sternberg SA, Schwartz AW, Karunananthan S, Bergman H, Clarfield AM. The identification of frailty: a systematic literature review. J Am Geriatr Soc. 2011;59(11):2129-2138.

7. Rockwood K, Mitnitski A. Frailty in relation to the accumulation of deficits. J Gerontol A Biol Sci Med Sci. 2007;62(7):722-727.

8. Kulminski AM, Ukraintseva SV, Kulminskaya IV, Arbeev KG, Land KC, Yashin AI. Cumulative deficits better characterize susceptibility to death in the elderly than phenotypic frailty: lessons from the cardiovascular health study. J Am Geriatr Soc. 2008;56(5):898-903.

9. Rizzoli R, Reginster J-Y, Arnal J-F, et al. Quality of life in sarcopenia and frailty. Calcif Tissue Int. 2013;93(2):101-120.

10. Collard RM, Boter H, Schoevers RA, Oude Voshaar RC. Prevalence of frailty in community-dwelling older persons: a systematic review. J Am Geriatr Soc. 2012;60(8):1487-1492.

11. Sirven N, Rapp T. The cost of frailty in France. Eur J Health Econ. 2017;18(2):243-253.

12. European Commission. Multidimensional Aspects of Population Ageing. Population Ageing in Europe: Facts, Implications and Policies: Outcomes of EU-funded Research. Luxembourg: Publications Office of the European Union; 2014.

13. Chen X, Mao G, Leng SX. Frailty syndrome: an overview. Clin Interv Aging. 2014;9:433-441.

14. Gill TM, Gahbauer EA, Allore HG, Han L. Transitions between frailty states among community-living older persons. Arch Intern Med. 2006; 166(4):418-423.

15. Xue QL. The frailty syndrome: definition and natural history. Clin Geriatr Med. 2011;27(1):1-15.

16. Cameron ID, Fairhall N, Gill L, et al. Developing interventions for frailty. Adv Geriatr. 2015;2015:7.

17. Gine-Garriga M, Roque-Figuls M, Coll-Planas L, Sitja-Rabert M, Salva A. Physical exercise interventions for improving performancebased measures of physical function in community-dwelling, frail older adults: a systematic review and meta-analysis. Arch Phys Med Rehabil. 2014;95(4):753.e3-769.e3.

18. Cadore EL, Rodriguez-Manas L, Sinclair A, Izquierdo M. Effects of different exercise interventions on risk of falls, gait ability, and balance in physically frail older adults: a systematic review. Rejuvenation Res. 2013;16(2):105-114.

19. Cermak NM, Res PT, de Groot LC, Saris WH, van Loon LJ. Protein supplementation augments the adaptive response of skeletal muscle to resistance-type exercise training: a meta-analysis. Am J Clin Nutr. 2012;96(6):1454-1464.

20. Fairhall N, Langron C, Sherrington C, et al. Treating frailty - a practical guide. BMC Med. 2011;9:83.

21. Vliek S, Melis RJ, Faes M, et al. Single versus multicomponent intervention in frail elderly: simplicity or complexity as precondition for success? J Nutr Health Aging. 2008;12(5):319-322.

22. Denison HJ, Cooper C, Sayer AA, Robinson SM. Prevention and optimal management of sarcopenia: a review of combined exercise and nutrition interventions to improve muscle outcomes in older people. Clin Interv Aging. 2015;10:859-869.

23. Daniels R, van Rossum E, de Witte L, Kempen G, van den Heuvel W. Interventions to prevent disability in frail community-dwelling elderly: a systematic review. BMC Health Serv Res. 2008;8:278.

24. Schneider N, Yvon C. A review of multidomain interventions to support healthy cognitive ageing. $J$ Nutr Health Aging. 2013;17(3): 252-257.

25. Bibas L, Levi M, Bendayan M, Mullie L, Forman DE, Afilalo J. Therapeutic interventions for frail elderly patients: part I. Published randomized trials. Prog Cardiovasc Dis. 2014;57(2):134-143.

26. Porter Starr KN, McDonald SR, Bales CW. Obesity and physical frailty in older adults: a scoping review of lifestyle intervention trials. $J \mathrm{Am}$ Med Dir Assoc. 2014;15(4):240-250.

27. Beswick AD, Rees K, Dieppe P, et al. Complex interventions to improve physical function and maintain independent living in elderly people: a systematic review and meta-analysis. Lancet. 2008; 371(9614):725-735.
28. Daniels R, Metzelthin S, van Rossum E, de Witte L, van den Heuvel W. Interventions to prevent disability in fail community-dwelling older persons: an overview. Eur J Ageing. 2010;7:37-55.

29. Lee PH, Lee YS, Chan DC. Interventions targeting geriatric frailty: a systemic review. J Clin Gerontol Geriatr. 2012;3(2):47-52.

30. Liberati A, Altman DG, Tetzlaff J, et al. The PRISMA statement for reporting systematic reviews and meta-analyses of studies that evaluate healthcare interventions: explanation and elaboration. $B M J$. 2009;339:b2700.

31. Slim K, Nini E, Forestier D, Kwiatkowski F, Panis Y, Chipponi J. Methodological index for non-randomized studies (minors): development and validation of a new instrument. ANZ J Surg. 2003;73(9):712-716.

32. Chan DC, Tsou HH, Yang RS, et al. A pilot randomized controlled trial to improve geriatric frailty. BMC Geriatr. 2012;12:58.

33. Chin A Paw MJ, de Jong N, Schouten EG, Hiddink GJ, Kok FJ. Physical exercise and/or enriched foods for functional improvement in frail, independently living elderly: a randomized controlled trial. Arch Phys Med Rehabil. 2001;82(6):811-817.

34. Chin A Paw MJ, de Jong N, Schouten EG, van Staveren WA, Kok FJ. Physical exercise or micronutrient supplementation for the wellbeing of the frail elderly? A randomised controlled trial [with consumer summary]. Br J Sports Med. 2002;36(2):126-131.

35. de Jong N. Sensible aging: using nutrient-dense foods and physical exercise with the frail elderly. Nutr Today. 2001;36(4):202-207.

36. de Jong N, Chin APMJ, de Groot LC, Hiddink GJ, van Staveren WA. Dietary supplements and physical exercise affecting bone and body composition in frail elderly persons. Am J Public Health. 2000;90(6):947-954.

37. de Jong N, Chin APMJ, de Graaf C, van Staveren WA. Effect of dietary supplements and physical exercise on sensory perception, appetite, dietary intake and body weight in frail elderly subjects. Br J Nutr. 2000; 83(6):605-613.

38. de Jong N, Chin APMJ, de Groot LC, de Graaf C, Kok FJ, van Staveren WA. Functional biochemical and nutrient indices in frail elderly people are partly affected by dietary supplements but not by exercise. J Nutr. 1999;129(11):2028-2036.

39. Chin A Paw MJ, de Jong N, Pallast EGM, Kloek GC, Schouten EG, KokFJ. Immunity in frail elderly: a randomized controlled trial of exercise and enriched foods. Med Sci Sports Exerc. 2000;32(12):2005-2011.

40. de Jong N, Chin APMJ, de Groot LC, et al. Nutrient-dense foods and exercise in frail elderly: effects on B vitamins, homocysteine, methylmalonic acid, and neuropsychological functioning. Am J Clin Nutr. 2001;73(2):338-346.

41. Hennessey JV, Chromiak JA, della Ventura S, et al. Growth hormone administration and exercise effects on muscle fiber type and diameter in moderately frail older people. $J$ Am Geriatr Soc. 2001;49(7): 852-858.

42. Kenny AM, Boxer RS, Kleppinger A, Brindisi J, Feinn R, Burleson JA. Dehydroepiandrosterone combined with exercise improves muscle strength and physical function in frail older women. $J$ Am Geriatr Soc. 2010;58(9):1707-1714.

43. Kim H, Suzuki T, Kim M, et al. Effects of exercise and milk fat globule membrane (MFGM) supplementation on body composition, physical function, and hematological parameters in community-dwelling frail Japanese women: a randomized double blind, placebo-controlled, follow-up trial. PLoS One. 2015;10(2):e0116256.

44. Kwon J, Yoshida Y, Yoshida H, Kim H, Suzuki T, Lee Y. Effects of a combined physical training and nutrition intervention on physical performance and health-related quality of life in prefrail older women living in the community: a randomized controlled trial. J Am Med Dir Assoc. 2015;16(3):261-268.

45. Ng TP, Feng L, Nyunt MS, et al. Nutritional, physical, cognitive, and combination interventions and frailty reversal among older adults: a randomized controlled trial. Am J Med. 2015;128(11):1225.e1-1236.e1.

46. Rydwik E, Lammes E, Frandin K, Akner G. Effects of a physical and nutritional intervention program for frail elderly people over age 75 . A randomized controlled pilot treatment trial. Aging Clin Exp Res. 2008; 20(2):159-170. 
47. Rydwik E, Frandin K, Akner G. Effects of a physical training and nutritional intervention program in frail elderly people regarding habitual physical activity level and activities of daily living - a randomized controlled pilot study. Arch Gerontol Geriatr. 2010;51(3):283-289.

48. Rydwik E, Gustafsson T, Frandin K, Akner G. Effects of physical training on aerobic capacity in frail elderly people (75+ years). Influence of lung capacity, cardiovascular disease and medical drug treatment: a randomized controlled pilot trial. Aging Clin Exp Res. 2010; 22(1):85-94.

49. Lammes E, Rydwik E, Akner G. Effects of nutritional intervention and physical training on energy intake, resting metabolic rate and body composition in frail elderly. A randomised, controlled pilot study. J Nutr Health Aging. 2012;16(2):162-167.

50. Tieland M, Dirks ML, van der Zwaluw N, et al. Protein supplementation increases muscle mass gain during prolonged resistance-type exercise training in frail elderly people: a randomized, double-blind, placebocontrolled trial. J Am Med Dir Assoc. 2012;13(8):713-719.

51. van de Rest O, van der Zwaluw NL, Tieland M, et al. Effect of resistance-type exercise training with or without protein supplementation on cognitive functioning in frail and pre-frail elderly: secondary analysis of a randomized, double-blind, placebo-controlled trial. Mech Ageing Dev. 2014;136-137:85-93.

52. Dorner TE, Lackinger C, Haider S, et al. Nutritional intervention and physical training in malnourished frail community-dwelling elderly persons carried out by trained lay "buddies": study protocol of a randomized controlled trial. BMC Public Health. 2013;13:1232.

53. Luger E, Dorner TE, Haider S, Kapan A, Lackinger C, Schindler K. Effects of a home-based and volunteer-administered physical training, nutritional, and social support program on malnutrition and frailty in older persons: a randomized controlled trial. J Am Med Dir Assoc. 2016;17(7):671.e9-671.e16.

54. Tarazona-Santabalbina F, Gomez-Cabrera M, Perez-Ros P, et al. A multicomponent exercise intervention that reverses frailty and improves cognition, emotion, and social networking in the communitydwelling frail elderly: a randomized clinical trial. J Am Med Dir Assoc. 2016;17(5):426-433.

55. Ikeda T, Aizawa J, Nagasawa H, et al. Effects and feasibility of exercise therapy combined with branched-chain amino acid supplementation on muscle strengthening in frail and pre-frail elderly people requiring long-term care: a crossover trial. Appl Physiol Nutr Metab. 2016;41(4):438-445.

56. de Labra C, Guimaraes-Pinheiro C, Maseda A, Lorenzo T, MillanCalenti JC. Effects of physical exercise interventions in frail older adults: a systematic review of randomized controlled trials. BMC Geriatr. 2015;15:154.

57. Malafarina V, Uriz-Otano F, Iniesta R, Gil-Guerrero L. Effectiveness of nutritional supplementation on muscle mass in treatment of sarcopenia in old age: a systematic review. J Am Med Dir Assoc. 2013;14(1): $10-17$.

58. Makanae Y, Fujita S. Role of exercise and nutrition in the prevention of sarcopenia. J Nutr Sci Vitaminol (Tokyo). 2015;61(Suppl):S125-S127.

59. Bosaeus I Rothenberg E. Nutrition and physical activity for the prevention and treatment of age-related sarcopenia. Proc Nutr Soc. 2016;75(2): 174-180.

60. Volpi E, Nazemi R, Fujita S. Muscle tissue changes with aging. Curr Opin Clin Nutr Metab Care. 2004;7(4):405-410.

61. Perkisas S, De Cock A, Verhoeven V, Vandewoude M. Physiological and architectural changes in the ageing muscle and their relation to strength and function in sarcopenia. Eur Geriatr Med. 2016;7(3):201-206.

62. Aguirre LE, Villareal DT. Physical exercise as therapy for frailty. Nestle Nutr Inst Workshop Ser. 2015;83:83-92.

63. Cadore EL, Casas-Herrero A, Zambom-Ferraresi A, et al. Multicomponent exercises including muscle power training enhance muscle mass, power output, and functional outcomes in institutionalized frail nonagenarians. Age (Dordr). 2014;36(2):773-785.

64. Rejeski WJ, Mihalko SL. Physical activity and quality of life in older adults. J Gerontol A Biol Sci Med Sci. 2001;56(Spec No 2):23-35.
65. Mura G, Carta MG. Physical activity in depressed elderly a systematic review. Clin Pract Epidemiol Mental Health. 2013;9:125-135.

66. Bherer L, Erickson KI, Liu-Ambrose T. A review of the effects of physical activity and exercise on cognitive and brain functions in older adults. J Aging Res. 2013;2013:8.

67. Theou O, Stathokostas L, Roland KP, et al. The effectiveness of exercise interventions for the management of frailty: a systematic review. J Aging Res. 2011;2011:569194.

68. Nash KCM. The effects of exercise on strength and physical performance in frail older people: a systematic review. Rev Clin Gerontol. 2012;22(4):274-285.

69. Edington J, Kon P, Martyn CN. Prevalence of malnutrition in patients in general practice. Clin Nutr. 1996;15(2):60-63.

70. Bonnefoy M, Berrut G, Lesourd B, et al. Frailty and nutrition: searching for evidence. J Nutr Health Aging. 2015;19(3):250-257.

71. Bartali B, Frongillo EA, Bandinelli S, et al. Low nutrient intake is an essential component of frailty in older persons. J Gerontol A Biol Sci Med Sci. 2006;61(6):589-593.

72. Deutz NE, Bauer JM, Barazzoni R, et al. Protein intake and exercise for optimal muscle function with aging: recommendations from the ESPEN Expert Group. Clin Nutr. 2014;33(6):929-936.

73. Bauer JM, Diekmann R. Protein supplementation with aging. Curr Opin Clin Nutr Metab Care. 2015;18(1):24-31.

74. Hickson M. Nutritional interventions in sarcopenia: a critical review. Proc Nutr Soc. 2015;74(4):378-386.

75. Bauer J, Biolo G, Cederholm T, et al. Evidence-based recommendations for optimal dietary protein intake in older people: a position paper from the PROT-AGE Study Group. J Am Med Dir Assoc. 2013; 14(8):542-559.

76. Wall B, Cermak N, Loon L. Dietary protein considerations to support active aging. Sports Med. 2014;44:185-194.

77. Kobayashi S, Asakura K, Suga H, Sasaki S. High protein intake is associated with low prevalence of frailty among old Japanese women: a multicenter cross-sectional study. Nutr J. 2013;12:164.

78. Gine-Garriga M, Guerra M, Pages E, Manini TM, Jimenez R, Unnithan VB. The effect of functional circuit training on physical frailty in frail older adults: a randomized controlled trial. J Aging Phys Act. 2010;18(4): 401-424.

79. Skelton DA, Young A, Greig CA, Malbut KE. Effects of resistance training on strength, power, and selected functional abilities of women aged 75 and older. J Am Geriatr Soc. 1995;43(10):1081-1087.

80. de Vries NM, van Ravensberg CD, Hobbelen JS, Olde Rikkert MG, Staal JB, Nijhuis-van der Sanden MW. Effects of physical exercise therapy on mobility, physical functioning, physical activity and quality of life in community-dwelling older adults with impaired mobility, physical disability and/or multi-morbidity: a meta-analysis. Ageing Res Rev. 2012;11(1):136-149.

81. Vagetti GC, Barbosa Filho VC, Moreira NB, Oliveira V, Mazzardo O, Campos W. Association between physical activity and quality of life in the elderly: a systematic review, 2000-2012. Rev Bras Psiquiatr. 2014;36(1):76-88.

82. Cameron ID, Fairhall N, Langron C, et al. A multifactorial interdisciplinary intervention reduces frailty in older people: randomized trial. BMC Med. 2013;11:65.

83. Fairhall N, Sherrington C, Kurrle SE, Lord SR, Lockwood K, Cameron ID. Effect of a multifactorial interdisciplinary intervention on mobilityrelated disability in frail older people: randomised controlled trial. BMC Med. 2012;10:120

84. Rockwood K, Song X, MacKnight C, et al. A global clinical measure of fitness and frailty in elderly people. CMAJ. 2005;173(5): 489-495.

85. Chan DC, Tsou HH, Chen CY, Chen CY. Validation of the ChineseCanadian study of health and aging clinical frailty scale (CSHA-CFS) telephone version. Arch Gerontol Geriatr. 2010;50(3):e74-e80.

86. Taylor HL, Jacobs DR Jr, Schucker B, Knudsen J, Leon AS, Debacker G. A questionnaire for the assessment of leisure time physical activities. J Chronic Dis. 1978;31(12):741-755. 
87. Chin A Paw MJ, Dekker JM, Feskens EJM, Schouten EG, Kromhout D. How to select a frail elderly population? A comparison of three working definitions. J Clin Epidemiol. 1999;52(11):1015-1021.

88. Mattiasson-Nilo I, Sonn U, Johannesson K, Gosman-Hedstrom G, Persson GB, Grimby G. Domestic activities and walking in the elderly: evaluation from a 30-hour heart rate recording. Aging (Milano). 1990; 2(2):191-198.

89. Frändin K, Grimby G. Assessment of physical activity, fitness and performance in 76-year-olds. Scand J Med Sci Sports. 1994;4(1): $41-46$.
90. Reuben DB, Siu AL. An objective measure of physical function of elderly outpatients. The Physical Performance Test. J Am Geriatr Soc. 1990;38(10):1105-1112.

91. Romero-Ortuno R, Walsh CD, Lawlor BA, Kenny RA. A frailty instrument for primary care: findings from the Survey of Health, Ageing and Retirement in Europe (SHARE). BMC Geriatr. 2010;10:57.

\section{Publish your work in this journal}

Clinical Interventions in Aging is an international, peer-reviewed journal focusing on evidence-based reports on the value or lack thereof of treatments intended to prevent or delay the onset of maladaptive correlates of aging in human beings. This journal is indexed on PubMed Central, MedLine,
CAS, Scopus and the Elsevier Bibliographic databases. The manuscript management system is completely online and includes a very quick and fair peer-review system, which is all easy to use. Visit http://www.dovepress. com/testimonials.php to read real quotes from published authors. 